\title{
A nonlocal phase-field model with nonconstant specific heat ${ }^{\dagger}$
}

\author{
PAVEL KREJČI $I^{\dagger}$ \\ Weierstrass Institute for Applied Analysis and Stochastics, \\ Mohrenstr. 39, D-10117 Berlin, Germany, and \\ Mathematical Institute, Czech Academy of Sciences, Žitná 25, CZ-11567 Praha 1, Czech Republic \\ ELISABETTA ROCCA ${ }^{\S}$ \\ Dipartimento di Matematica, Università di Milano, Via Saldini 50, 20133 Milano, Italy \\ AND \\ JÜRGEN SPREKELS ${ }^{\text {II }}$ \\ Weierstrass Institute for Applied Analysis and Stochastics, \\ Mohrenstr. 39, D-10117 Berlin, Germany
}

[Received 26 April 2006 and in revised form 17 January 2007]

\begin{abstract}
We prove the existence, uniqueness, thermodynamic consistency, global boundedness from both above and below, and continuous data dependence for a solution to an integrodifferential model for nonisothermal phase transitions under nonhomogeneous mixed boundary conditions. The specific heat is allowed to depend on the order parameter, and the convex component of the free energy may or may not be singular.
\end{abstract}

2000 Mathematics Subject Classification: 35B50, 35B65, 35K50, 45J05, 45K05.

Keywords: Phase transitions; nonlocal models; integrodifferential equations.

\section{Introduction}

Phase-field models have been designed to describe the evolution of the state variables $\theta>0$ and $\chi$, representing the absolute temperature and a scalar order parameter, respectively, during temperatureinduced phase transitions in a body $\Omega \subset \mathbb{R}^{N}(N=3$, for instance) if no mechanical motion takes place. For example, in a simple melting-solidification process, $\chi$ attains its values in the interval $[0,1]$, where $\{\chi=0\}$ characterizes the solid phase, $\{\chi=1\}$ the liquid, and $\{0<\chi<1\}$ is the liquid fraction in a mixture of both phases. Solid-solid phase transitions between two crystallographic variants with different mechanical properties (martensite and austenite, say) may also exhibit a similar behavior provided the experiment is uniaxial and is carried out under constant strain. Then the stress may play the role of an order parameter characterizing the phase, but no natural restriction on the admissible order parameter range is necessary.

\footnotetext{
${ }^{\dagger}$ Supported by the DFG Research Center MATHEON.

E-mail: krejci@wias-berlin.de, krejci@math.cas.cz

${ }^{\S}$ A large part of this work was done during E. Rocca's visit at WIAS Berlin in November 2005. E-mail: rocca@mat.unimi.it

II E-mail: sprekels@wias-berlin.de
} 
We deal here with an integrodifferential model for volume preserving nonisothermal phase transitions that takes into account long-range interactions between particles. The physical relevance of nonlocal interaction phenomena in phase separation and phase transition models was already described in the pioneering papers [28] and [8]; however, only recently both isothermal and nonisothermal models containing nonlocal terms have been analyzed in a more systematic way (cf., e.g., [2]-[3], [9]-[21], [26]). The difference between local and nonlocal models consists in a different choice of the particle interaction potential in the free energy functional. The nonlocal contribution to the free energy has typically the form $\int_{\Omega} k(x, y)|\chi(x)-\chi(y)|^{2} \mathrm{~d} y$ with a given symmetric kernel $k(x, y)$; its classical local Ginzburg-Landau counterpart has the form $(v / 2)|\nabla \chi(x)|^{2}$ as, e.g., in [7], with a positive parameter $v$, and can be obtained as a formal limit as $m \rightarrow \infty$ from the nonlocal one with the choice $k(x, y)=m^{N+2} K\left(|m(x-y)|^{2}\right)$, where $K$ is a nonnegative function with support in $[0,1]$. This follows from the formula

$$
\begin{aligned}
\int_{\Omega} m^{N+2} K\left(|m(x-y)|^{2}\right)|\chi(x)-\chi(y)|^{2} \mathrm{~d} y & =\int_{\Omega_{m}(x)} K\left(|z|^{2}\right)\left|\frac{\chi(x+z / m)-\chi(x)}{1 / m}\right|^{2} \mathrm{~d} z \\
& \stackrel{m \rightarrow \infty}{\longrightarrow} \int_{\mathbb{R}^{N}} K\left(|z|^{2}\right)\langle\nabla \chi(x), z\rangle^{2} \mathrm{~d} z=\frac{v}{2}|\nabla \chi(x)|^{2}
\end{aligned}
$$

for a sufficiently regular $\chi$, where $v=(2 / N) \int_{\mathbb{R}^{N}} K\left(|z|^{2}\right)|z|^{2} \mathrm{~d} z$ and $\Omega_{m}(x)=m(\Omega-x)$. We have used the identity $\int_{\mathbb{R}^{N}} K\left(|z|^{2}\right)\langle e, z\rangle^{2} \mathrm{~d} z=(1 / N) \int_{\mathbb{R}^{N}} K\left(|z|^{2}\right)|z|^{2} \mathrm{~d} z$ for every unit vector $e \in \mathbb{R}^{N}$. Let us also mention the "Penrose-Fife" potential $(\nu / 2) \theta|\nabla \chi(x)|^{2}$ (see [6, 25]). Its nonlocal version might also deserve appropriate attention (cf. [21]), but we do not consider this issue here.

The passage from a nonlocal to a local potential changes drastically the properties of the model. For example, the maximum principle is lost in the limit, and in general it is not possible to guarantee without additional hypotheses that the absolute temperature remains positive during the process.

We pursue here the investigations initiated in [19] and consider a local free energy of the form

$$
F[\theta, \chi]=c_{V}(\chi) \theta(1-\log \theta)+\theta \sigma(\chi)+\lambda(\chi)+(\beta+\theta) \varphi(\chi)+B[\chi],
$$

where $\sigma$ and $\lambda$ are smooth functions describing the local dependence on $\chi$ of the entropy and of the latent heat, respectively; $\beta>0$ is a constant parameter, $B[\chi]$ is a nonlocal operator of the form

$$
B[\chi](x, t):=\int_{\Omega} k(x, y) G(\chi(x, t)-\chi(y, t)) \mathrm{d} y
$$

with a bounded, symmetric kernel $k: \Omega \times \Omega \rightarrow \mathbb{R}$ and an even smooth function $G ; \varphi$ is a general proper, convex, and lower semicontinuous function. Its domain $\mathcal{D}(\varphi)$ may be bounded or unbounded, depending on the specific model situation. The main novelty here is that the specific heat $c_{V}$ may depend on the order parameter $\chi$. In the solid-liquid system mentioned above, for example, we may have different values $c_{V}^{0}$ in the solid and $c_{V}^{1}$ in the liquid. Assuming that their dependence on temperature can be neglected in each phase, we may define $c_{V}(\chi)=c_{V}^{0}+\chi\left(c_{V}^{1}-c_{V}^{0}\right)$ (cf. [27, Section IV.4]). The value of $\chi$ can be kept between 0 and 1 by setting in this case $\varphi=I_{[0,1]}$ (the indicator function of $[0,1])$.

With the above free energy, we associate the local internal energy $E$ and entropy $S$ according to the formulas

$$
S=-\frac{\partial F}{\partial \theta}, \quad E=F+\theta S,
$$


that is,

$$
\left\{\begin{array}{l}
S[\theta, \chi]=c_{V}(\chi) \log \theta-\sigma(\chi)-\varphi(\chi), \\
E[\theta, \chi]=c_{V}(\chi) \theta+\lambda(\chi)+\beta \varphi(\chi)+B[\chi] .
\end{array}\right.
$$

The temperature dynamics is governed by the internal energy balance over an arbitrary control volume $\Omega^{\prime} \subset \Omega$,

$$
\frac{\mathrm{d}}{\mathrm{d} t} \int_{\Omega^{\prime}} E[\theta, \chi] \mathrm{d} x+\int_{\partial \Omega^{\prime}}\langle\mathbf{q}, \mathbf{n}\rangle \mathrm{d} s=\Psi\left(\Omega^{\prime}\right),
$$

where $\mathbf{q}$ is the heat flux vector, $\mathbf{n}$ is the unit outward normal to $\partial \Omega^{\prime}$, and $\Psi\left(\Omega^{\prime}\right)$ is the energy exchange through the boundary of $\Omega^{\prime}$ due to the nonlocal interactions. The order parameter dynamics is assumed in the form

$$
\mu(\theta) \chi_{t} \in-\delta_{\chi} \mathcal{F}[\theta, \chi]
$$

with a factor $\mu(\theta)>0$, where we denote

$$
\mathcal{F}[\theta, \chi]=\int_{\Omega} F[\theta, \chi] \mathrm{d} x,
$$

and where $\delta_{\chi} \mathcal{F}$ stands for the variational derivative of $\mathcal{F}$ with respect to the variable $\chi$. The inclusion sign in (1.6) accounts for the fact that $\mathcal{F}$ may contain components that are not Fréchet differentiable, but are convex, and the derivative can be interpreted as the subdifferential, which may be multivalued. Condition $(1.6)$ is based on the assumption that the system tends to move towards local minima of the free energy with a speed proportional to $1 / \mu(\theta)$. Using [1.1, we can rewrite 1.6 as

$$
\mu(\theta) \chi_{t}+c_{V}^{\prime}(\chi) \theta(1-\log \theta)+\lambda^{\prime}(\chi)+\theta \sigma^{\prime}(\chi)+(\beta+\theta) \partial \varphi(\chi)+b[\chi] \ni 0,
$$

with the notation

$$
b[\chi](x, t):=2 \int_{\Omega} k(x, y) G^{\prime}(\chi(x, t)-\chi(y, t)) \mathrm{d} y .
$$

The interaction term $\Psi\left(\Omega^{\prime}\right)$ in 1.5 and the constitutive law for the heat flux have to comply with the Clausius-Duhem inequality

$$
S_{t}+\operatorname{div}\left(\frac{\mathbf{q}}{\theta}\right) \geqslant 0
$$

which is understood here almost everywhere in the regularity context of Theorem 2.2 Assuming $\theta>0$ (this will have to be justified in the next sections), and using (1.1) with 1.7 ) and (1.3), we obtain the identities

$$
\begin{aligned}
\theta\left(S_{t}+\operatorname{div}\left(\frac{\mathbf{q}}{\theta}\right)\right) & =E_{t}+\operatorname{div} \mathbf{q}-F_{t}-\theta_{t} S-\frac{\langle\mathbf{q}, \nabla \theta\rangle}{\theta} \\
& =E_{t}+\operatorname{div} \mathbf{q}+\mu(\theta) \chi_{t}^{2}-\frac{\langle\mathbf{q}, \nabla \theta\rangle}{\theta}+b[\chi] \chi_{t}-B[\chi]_{t} .
\end{aligned}
$$

We assume the Fourier heat flux law

$$
\mathbf{q}=-\kappa \nabla \theta,
$$


with a constant positive heat conductivity $\kappa$. Then the right-hand side of $[1.10]$ stays nonnegative without prescribing any relationship between $\mu(\theta)$ and $B[\chi]$, provided that we choose $\Psi\left(\Omega^{\prime}\right)$ in 1.5 as

$$
\Psi\left(\Omega^{\prime}\right)=\int_{\Omega^{\prime}}\left(-b[\chi] \chi_{t}+B[\chi]_{t}\right) \mathrm{d} x .
$$

In agreement with natural expectations, we have $\Psi(\Omega)=0$. The differential form of the energy balance $(1.5)$ then reads

$$
E_{t}+\operatorname{div} \mathbf{q}=-b[\chi] \chi_{t}+B[\chi]_{t}
$$

that is,

$$
\left(c_{V}(\chi) \theta+\lambda(\chi)+\beta \varphi(\chi)\right)_{t}+b[\chi] \chi_{t}-\kappa \Delta \theta=0 .
$$

In real materials, the dependence of $c_{V}$ on the phase may be very strong (the specific heat in water is considerably higher than both in ice and in vapor, for instance). Introducing the term $c_{V}(\chi)$ into the above system may however create substantial difficulties from both the physical and mathematical viewpoints, which can again be illustrated on the two-phase system mentioned above. More specifically, consider the thermodynamically insulated (i.e., with homogeneous Neumann boundary conditions) relaxed Stefan problem corresponding to the choice $\varphi=I_{[0,1]}, \lambda^{\prime}(\chi)=L$, $\sigma^{\prime}(\chi)=-L / \theta_{c}, B[\chi] \equiv 0$, where $L$ and $\theta_{c}$ are positive constants (the latent heat and phase transition temperature, respectively), $c_{V}^{\prime}(\chi)=\bar{c}:=c_{V}^{1}-c_{V}^{0}$. Thermodynamic equilibria are located on the curve

$$
\partial I_{[0,1]}(\chi) \ni \bar{c} \theta(\log \theta-1)+\frac{L}{\theta_{c}}\left(\theta-\theta_{c}\right),
$$

or, equivalently,

$$
\chi \in \mathfrak{H}\left(\bar{c} \theta(\log \theta-1)+\frac{L}{\theta_{c}}\left(\theta-\theta_{c}\right)\right),
$$

where $\mathfrak{H}$ is the maximal monotone extension of the Heaviside function. We see that if $c_{V}^{1}<c_{V}^{0}$, as in the water-vapor system, then the only (stable!) equilibrium for both very high and very low temperatures is $\chi=0$, which is an obvious physical paradox. Between water and ice, this contradiction does not occur.

We focus here on mathematical problems arising in connection with this model. On the boundary of $\Omega$, we prescribe nonhomogeneous mixed boundary conditions. Our main results include the proof of existence and uniqueness of a global solution $(\theta, \chi)$ to 1.7 and 1.13 on the whole time axis $(0, \infty)$. We also prove that $\theta$ is uniformly bounded from above and below on $(0, \infty)$, with the intention to study the asymptotic behavior $t \rightarrow \infty$ in the future. Note that there are only few works in the literature dealing with the convergence of trajectories towards equilibrium for nonlocal phase-field systems. The case of analytic potentials $\varphi$ has been solved first in [11] and then in [17] for a time-relaxed model and in [13] for a time-discrete scheme. The nonsmooth case is not straightforward even if the nonlocal term is absent (see [23]), and deserves special attention.

The paper is organized as follows. The main results are stated in Section 2 Section 3 is devoted to some auxiliary results on a class of differential inclusions, on maximum principles for parabolic equations with nonconstant coefficients and nonhomogeneous mixed boundary conditions, and on $L^{\infty}$-estimates based on Moser-type iterations. Uniqueness is proved in Section 4 , existence and global boundedness in time in Section 5 


\section{Main results}

Consider a bounded domain $\Omega \subset \mathbb{R}^{N}, N \geqslant 1$, and the time interval $[0, \infty)$. For $T \in(0, \infty](\infty$ included) we denote by $Q_{T}=\Omega \times(0, T)$ the open space-time cylinder, and by $\Sigma_{T}$ its lateral boundary $\partial \Omega \times(0, T)$. We use, for the sake of simplicity, the same symbol $H$ for both $L^{2}(\Omega)$ and $L^{2}\left(\Omega ; \mathbb{R}^{N}\right)$, and $H^{1}$ for $H^{1}(\Omega)$.

We rewrite system (1.13), (1.7), putting, for simplicity and without loss of generality, $\kappa=1$, in the form

$$
\begin{aligned}
& \left(c_{V}(\chi) \theta+\lambda(\chi)+\beta \varphi(\chi)\right)_{t}+b[\chi] \chi_{t}-\Delta \theta=0, \\
& \mu(\theta) \chi_{t}+c_{V}^{\prime}(\chi) \theta(1-\log \theta)+\lambda^{\prime}(\chi)+\theta \sigma^{\prime}(\chi)+(\beta+\theta) \partial \varphi(\chi)+b[\chi] \ni 0,
\end{aligned}
$$

to be satisfied in a sense specified below, with $b[\chi]$ defined in $[1.8$, and prescribe the boundary and initial conditions

$$
\begin{array}{ll}
\partial_{\mathbf{n}} \theta+\gamma\left(\theta-\theta_{\Gamma}\right)=0 & \text { on } \Sigma_{\infty}, \\
\theta(0)=\theta_{0}, \quad \chi(0)=\chi_{0} & \text { in } \Omega,
\end{array}
$$

where $\partial_{\mathbf{n}}$ denotes the outward normal derivative, and the data fulfil the following hypothesis.

Hypothesis 2.1 We fix positive constants $\beta, c_{0}, \bar{\theta}_{\Gamma}, \mu_{*}, C_{0}, \theta_{*}$, and assume that:

(i) $\gamma \in L^{\infty}(\partial \Omega)$ is a nonnegative function.

(ii) There exist constants $\psi^{*}>\psi_{*}>0$ such that $\psi^{*} \geqslant \psi_{1}(x) \geqslant \psi_{*}$ a.e., where $\psi_{1} \in H^{1}$ is the eigenfunction with unit $H$-norm corresponding to the smallest eigenvalue $\lambda_{1} \geqslant 0$ of the elliptic problem

$$
-\Delta \psi_{1}=\lambda_{1} \psi_{1} \quad \text { in } \Omega, \quad \partial_{\mathbf{n}} \psi_{1}+\gamma \psi_{1}=0 \quad \text { on } \partial \Omega
$$

(iii) $\varphi: \mathbb{R} \rightarrow[0, \infty]$ is a proper, convex, and lower semicontinuous function, $\mathcal{D}(\varphi)$ is its domain, and $0 \in \partial \varphi(0)$.

(iv) $\sigma, \lambda \in W^{2, \infty}(\mathcal{D}(\varphi))$.

(v) $G \in W^{2, \infty}(\mathcal{D}(\varphi)-\mathcal{D}(\varphi)), G(z)=G(-z)$ for all $z \in(\mathcal{D}(\varphi)-\mathcal{D}(\varphi)), k \in L^{\infty}(\Omega \times \Omega)$, $k(x, y)=k(y, x)$ a.e. in $\Omega \times \Omega$.

(vi) $c_{V} \in W^{2, \infty}(\mathcal{D}(\varphi)), c_{V}(z) \geqslant c_{0}>0$ for all $z \in \mathcal{D}(\varphi)$.

(vii) $\theta_{\Gamma} \in L^{\infty}\left(\Sigma_{\infty}\right),\left(\theta_{\Gamma}\right)_{t} \in L_{\mathrm{loc}}^{2}\left(\Sigma_{\infty}\right), \theta_{\Gamma} \geqslant 0$ a.e. in $\Sigma_{\infty}$.

(viii) $\mu$ is locally Lipschitz in $\mathbb{R}^{+}, \mu(\tau) \geqslant \mu_{*}(1+\tau)$ for all $\tau \in \mathbb{R}^{+}$.

(ix) For any $C>0$ set $\mathcal{D}_{C}(\varphi)=\{\chi \in \mathcal{D}(\varphi) ; \partial \varphi(\chi) \cap[-C, C] \neq \emptyset\}$, and assume that $\chi_{0} \in$ $L^{\infty}(\Omega), \chi_{0}(x) \in \mathcal{D}_{C_{0}}(\varphi)$ a.e. in $\Omega$.

(x) $\theta_{0} \in H^{1} \cap L^{\infty}(\Omega), \theta_{0}(x) \geqslant \theta_{*}$ a.e. in $\Omega$.

If $\gamma$ vanishes on some part of $\partial \Omega$, then $(2.3)$ is a mixed Neumann-Robin boundary condition.

Below in Remark 2.3 we will show some sufficient conditions for Hypothesis 2.1 (ii) to hold. Note also that by [5, Example 2.3.4], $\partial \varphi$ is maximal monotone, hence $\mathcal{D}_{C}(\varphi)$ is a closed (possibly unbounded or degenerate) interval for every $C>0$.

We are in a position to state the existence theorem. 
TheOREM 2.2 Let Hypothesis 2.1 hold. Then there exists at least one pair $(\theta, \chi)$ with the regularity

$$
\begin{aligned}
& \theta \in L^{\infty}\left(Q_{\infty}\right) \cap L^{\infty}\left(0, \infty ; H^{1}\right), \quad \theta_{t} \in L_{\mathrm{loc}}^{2}\left(0, \infty ; L^{2}(\Omega)\right), \\
& \theta(x, t)>0 \quad \text { a.e. in } Q_{\infty}, \\
& \chi \in L_{\mathrm{loc}}^{\infty}\left(Q_{\infty}\right), \quad \chi_{t} \in L^{\infty}\left(Q_{\infty}\right) ; \quad \exists C>0: \chi(x, t) \in \mathcal{D}_{C}(\varphi) \quad \text { a.e. in } Q_{\infty},
\end{aligned}
$$

satisfying 2.2), 2.4 a.e., together with

$\int_{\Omega}\left(\left(c_{V}(\chi) \theta+\lambda(\chi)+\beta \varphi(\chi)\right)_{t}+b[\chi] \chi_{t}\right) w \mathrm{~d} x+\int_{\Omega}\langle\nabla \theta, \nabla w\rangle \mathrm{d} x+\int_{\partial \Omega} \gamma\left(\theta-\theta_{\Gamma}\right) w \mathrm{~d} s=0$,

for every test function $w \in H^{1}$. Moreover, there exist two positive constants $\bar{\theta}$ and $\underline{\theta}$ (independent of $t$ ) such that the following uniform upper and lower bounds hold:

$$
\underline{\theta}<\theta(x, t)<\bar{\theta} \quad \text { for a.e. }(x, t) \in Q_{\infty} .
$$

REMARK 2.3 Hypothesis 2.1 (ii) is fulfilled for example if $\gamma \equiv 0$. Then $\lambda_{1}=0$ and $\psi_{1}$ is a constant function. Another easy case is when $\Omega=\left(a_{1}, b_{1}\right) \times \cdots \times\left(a_{N}, b_{N}\right)$ is an orthogonal parallelepiped with $\gamma$ constant on each side. As a last example, let us mention the case where both $\partial \Omega$ and $\gamma$ are of class $C^{\infty}$. The first eigenfunction $\psi_{1}$ is defined as a minimizer of the Rayleigh functional

$$
\mathcal{R}(u)=\int_{\Omega}|\nabla u|^{2} \mathrm{~d} x+\int_{\partial \Omega} \gamma u^{2} \mathrm{~d} s
$$

on the set $S_{1}^{1}:=\left\{u \in H^{1} ;|u|_{H}=1\right\}$, and $\lambda_{1}$ is given as $\lambda_{1}=\min \left\{\mathcal{R}(u) ; u \in S_{1}^{1}\right\}$. The function $\psi_{1}$ thus satisfies the variational equation

$$
\int_{\Omega}\left\langle\nabla \psi_{1}, \nabla w\right\rangle \mathrm{d} x+\int_{\partial \Omega} \gamma \psi_{1} w \mathrm{~d} s=\lambda_{1} \int_{\Omega} \psi_{1} w \mathrm{~d} x
$$

for every $w \in H^{1}$. Choosing $w=\psi_{1}^{+}, w=\psi_{1}^{-}$(the positive and negative parts of $\psi_{1}$, respectively), we see that both $\psi_{1}^{+}, \psi_{1}^{-}$, as well as $\left|\psi_{1}\right|=\psi_{1}^{+}+\psi_{1}^{-}$, satisfy the variational equation 2.11 . Then $\left|\psi_{1}\right|$ is a weak solution of the problem

$$
-\Delta\left|\psi_{1}\right|=\lambda_{1}\left|\psi_{1}\right| \quad \text { in } \Omega, \quad \partial_{\mathbf{n}}\left|\psi_{1}\right|+\gamma\left|\psi_{1}\right|=0 \quad \text { on } \partial \Omega .
$$

By [24, Chap. 2, Thm. 5.1], we have, denoting $H^{r}=W^{r, 2}(\Omega), H^{0}=H$, that

$$
|| \psi_{1}||_{H^{2+r}} \leqslant C_{r}\left(\left|\lambda_{1}\right| \psi_{1}||_{H^{r}}+|| \psi_{1}||_{H^{1+r}}\right)
$$

for every integer $r \geqslant 0$ with some constants $C_{r}>0$ provided the right-hand side is well defined. This is the case for $r=0$, hence we may iterate in 2.13 for $r=1,2, \ldots$ until we obtain $\left|\psi_{1}\right| \in$ $C^{2}(\bar{\Omega})$ taking $r$ sufficiently large. The function $\left|\psi_{1}\right|$ does not vanish in $\Omega$, by Maximum Principle I in [4, Part II, Chap. 2]. Assuming that there exists $x \in \partial \Omega$ such that $\left|\psi_{1}(x)\right|=0$ also leads to a contradiction. Indeed, we find a ball lying entirely in $\Omega$ and touching $\partial \Omega$ at the point $x$. Maximum Principle III in [4, Part II, Chap. 2] then shows that $\partial_{\mathbf{n}}\left(\left|\psi_{1}(x)\right|\right)<0$, which contradicts (2.12). Hence $\psi_{1}$ does not vanish in $\bar{\Omega}$. This argument also shows that this eigenfunction of problem $(2.5)$ is unique up to a constant multiple. 
To conclude this section, we state a result on uniqueness and continuous data dependence for (2.1.2.4).

THEOREM 2.4 Let Hypothesis 2.1 hold, and let $\left(\theta_{1}, \chi_{1}\right),\left(\theta_{2}, \chi_{2}\right)$ be solutions to 2.12 2.4 in the sense of Theorem 2.2 associated with boundary and initial data $\theta_{\Gamma 1}, \theta_{01}, \chi_{01}$ and $\theta_{\Gamma 2}, \theta_{02}, \chi_{02}$, respectively. Put $\hat{\theta}=\theta_{1}-\theta_{2}, \hat{\chi}=\chi_{1}-\chi_{2}, \hat{\theta}_{\Gamma}=\theta_{\Gamma 1}-\theta_{\Gamma 2}, \hat{\chi}_{0}=\chi_{01}-\chi_{02}, \hat{\theta}_{0}=\theta_{01}-\theta_{02}$. Then for every $T>0$ there exists a constant $C_{T}>0$ such that

$$
\begin{aligned}
\int_{0}^{T} \int_{\Omega}|\hat{\theta}(x, t)|^{2} \mathrm{~d} x \mathrm{~d} t+\max _{t \in[0, T]} & \int_{\Omega}|\hat{\chi}(x, t)|^{2} \mathrm{~d} x \\
& \leqslant C_{T}\left(\left|\hat{\theta}_{0}\right|_{H}^{2}+\left|\hat{\chi}_{0}\right|_{H}^{2}+\int_{0}^{T} \int_{\partial \Omega} \gamma \hat{\theta}_{\Gamma}^{2}(x, t) \mathrm{d} s \mathrm{~d} t\right) .
\end{aligned}
$$

\section{Auxiliary results}

In this section we provide some auxiliary results that are used in the remainder of the paper. The first part of this section deals with the continuity of solution operators to general differential inclusions, while the second one recalls some parabolic maximum principle results and a variant of the Moser iteration scheme.

\subsection{Solution operators to differential inclusions}

Consider a functional $\varphi$ as in Hypothesis 2.1 (iii). For a given initial condition $\chi_{0}$, a fixed final time $T>0$, and a given function $\theta \in L^{1}\left(Q_{T}\right)$, we solve the following differential inclusion:

$$
\alpha(\theta) \chi_{t}+\partial \varphi(\chi) \ni f[\chi, \theta] \quad \text { a.e. in } Q_{T}, \quad \chi(x, 0)=\chi_{0}(x) \quad \text { a.e. in } \Omega,
$$

where $\alpha: \mathbb{R} \rightarrow \mathbb{R}$ is a given function, and $f: L^{1}\left(Q_{T}\right) \times L^{1}\left(Q_{T}\right) \rightarrow L^{1}\left(Q_{T}\right)$ is a given continuous operator satisfying the following hypothesis.

HyPOTHESIS 3.1 There exist positive constants $\alpha_{0}, L, C$ such that:

(i) $\alpha_{0} \leqslant \alpha(\theta)$ for all $\theta \in \mathbb{R}$.

(ii) $\left|\alpha\left(\theta_{1}\right)-\alpha\left(\theta_{2}\right)\right| \leqslant L\left|\theta_{1}-\theta_{2}\right|$ for all $\theta_{1}, \theta_{2} \in \mathbb{R}$.

(iii) $|f[\chi, \theta](x, t)| \leqslant C$ a.e. in $Q_{T}$ for all $\chi, \theta \in L^{1}\left(Q_{T}\right)$ such that $\chi(x, t) \in \mathcal{D}(\varphi)$ a.e. in $Q_{T}$.

(iv) $\left|f\left[\chi_{1}, \theta\right]-f\left[\chi_{2}, \theta\right]\right|_{L^{1}\left(Q_{t}\right)} \leqslant L\left|\chi_{1}-\chi_{2}\right|_{L^{1}\left(Q_{t}\right)}$ for all $\chi_{1}, \chi_{2}, \theta \in L^{1}\left(Q_{T}\right)$ and $t \in[0, T]$.

This is slightly different from [19, Subsection 3.1], where $f$ is assumed to be Lipschitz continuous also with respect to $\theta$. Here, $f$ is only continuous, and we therefore only get the following weaker result.

Proposition 3.2 Let Hypothesis 3.1 hold, and let $\mathcal{D}_{C}(\varphi)$ be as in Hypothesis 2.1 Then, for every $\theta \in L^{1}\left(Q_{T}\right)$, and for every $\chi_{0} \in L^{\infty}(\Omega)$ with $\chi_{0}(x) \in \mathcal{D}_{C}(\varphi)$ a.e. in $\Omega$, there exists a unique solution $\chi \in L^{\infty}\left(Q_{T}\right)$ to 3.1 such that $\chi_{t} \in L^{\infty}\left(Q_{T}\right)$, and we have

$$
\chi(x, t) \in \mathcal{D}_{C}(\varphi), \quad\left|f[\chi, \theta](x, t)-\alpha(\theta(x, t)) \chi_{t}(x, t)\right| \leqslant C \quad \text { a.e. in } Q_{T} .
$$

Moreover, let $\left\{\theta^{(n)}\right\}$ be a sequence that converges strongly in $L^{1}\left(Q_{T}\right)$ to $\theta$, let $\chi_{0}^{(n)} \in L^{\infty}(\Omega)$ be initial conditions such that $\chi_{0}^{(n)}(x) \in \mathcal{D}_{C}(\varphi)$ a.e. in $\Omega$ and $\chi_{0}^{(n)} \rightarrow \chi_{0}$ in $L^{1}(\Omega)$, and let $\chi^{(n)}, \chi$ be the respective solutions to 3.1 . Then $\chi^{(n)} \rightarrow \chi, \chi_{t}^{(n)} \rightarrow \chi_{t}$ strongly in $L^{1}\left(Q_{T}\right)$. 
REMARK 3.3 As a complement to the above proposition, notice that the strong continuity $L^{1}\left(Q_{T}\right) \rightarrow L^{p}\left(Q_{T}\right)$ of the solution mapping for $1 \leqslant p<\infty$ follows from the uniform $L^{\infty}$-bound 3.2. Indeed, testing 3.1 by $\chi_{t}$, we obtain the identity

$$
\varphi(\chi)_{t}=-\alpha(\theta) \chi_{t}^{2}+f[\chi, \theta] \chi_{t} \quad \text { a.e. in } Q_{T} .
$$

If $\theta^{(n)}, \theta, \chi^{(n)}, \chi$ are as in Proposition 3.2 the $L^{\infty}$-bounds 3.2 show that $\chi^{(n)} \rightarrow \chi, \chi_{t}^{(n)} \rightarrow \chi_{t}$, $\varphi\left(\chi^{(n)}\right)_{t} \rightarrow \varphi(\chi)_{t}$, strongly in any $L^{p}\left(Q_{T}\right)$ for $1 \leqslant p<\infty$.

The proof of Proposition 3.2 is based on properties of the corresponding space-independent problem. For a given initial condition $\chi_{0} \in \mathcal{D}(\varphi)$ and a given function $\theta \in L^{1}(0, T)$, we consider the differential inclusion

$$
\alpha(\theta(t)) \dot{\chi}(t)+\partial \varphi(\chi(t)) \ni g(t) \quad \text { a.e. in }(0, T), \quad \chi(0)=\chi_{0},
$$

where $\alpha: \mathbb{R} \rightarrow \mathbb{R}$ is as in Hypothesis 3.1 and $g \in L^{\infty}(0, T)$ is such that

$$
|g(t)| \leqslant C \quad \text { a.e. in }(0, T) .
$$

We recall from [19, Proposition 3.4] the following result.

Proposition 3.4 Let Hypotheses 3.1 (i)-(ii) and 3.5 hold. Then, for every $\theta \in L^{1}(0, T)$ and every $\chi_{0} \in \mathcal{D}_{C}(\varphi)$, there exists a unique solution $\chi \in W^{1, \infty}(0, T)$ to 3.4$)$, and we have

$$
\chi(t) \in \mathcal{D}_{C}(\varphi) \quad \forall t \in[0, T], \quad|g(t)-\alpha(\theta(t)) \dot{\chi}(t)| \leqslant C \quad \text { a.e. in }(0, T) .
$$

Moreover, there exists a positive constant $C^{\#}$ depending only on $C, \alpha_{0}$, and $L$ such that the solutions $\chi_{1}, \chi_{2} \in W^{1, \infty}(0, T)$ associated with $\chi_{01}, \chi_{02} \in \mathcal{D}_{C}(\varphi), \theta_{1}, \theta_{2} \in L^{1}(0, T)$, and $g_{1}, g_{2} \in L^{\infty}(0, T)$ with the constraint (3.5), satisfy the inequality

$$
\left|\dot{\chi}_{1}-\dot{\chi}_{2}\right|(t)+\frac{\mathrm{d}}{\mathrm{d} t}\left|\chi_{1}-\chi_{2}\right|(t) \leqslant C^{\#}\left(\left|\theta_{1}-\theta_{2}\right|(t)+\left|g_{1}-g_{2}\right|(t)\right) \quad \text { a.e. in }(0, T) \text {. }
$$

We now use 3.7 to prove the convergence statement in Proposition 3.2

Proof of Proposition 3.2. For given $\theta \in L^{1}\left(Q_{T}\right)$ and $\chi_{0} \in L^{\infty}(\Omega)$ with $\chi_{0}(x) \in \mathcal{D}_{C}(\varphi)$ a.e., we obtain the existence of a unique solution to (3.1) by the Banach contraction argument in the same way as in the proof of [19. Proposition 3.2]. To prove the continuity of the solution mapping, consider the sequences $\chi_{0}^{(n)}, \theta^{(n)}, \chi^{(n)}$ as above. For almost all $x \in \Omega$, we use 3.7) with $\theta_{1}(t)=$ $\theta(x, t), \theta_{2}(t)=\theta^{(n)}(x, t), \chi_{1}(t)=\chi(x, t), \chi_{2}(t)=\chi^{(n)}(x, t), g_{1}(t)=f[\chi, \theta](x, t), g_{2}(t)=$ $f\left[\chi^{(n)}, \theta^{(n)}\right](x, t)$. Integrating over $\Omega \times(0, t)$ for $t \in(0, T]$, and using Hypothesis 3.1 we obtain

$$
\begin{aligned}
\int_{0}^{t} \int_{\Omega}\left|\chi_{t}-\chi_{t}^{(n)}\right|(x, s) \mathrm{d} x \mathrm{~d} s+\int_{\Omega} \mid \chi & -\chi^{(n)}|(x, t) \mathrm{d} x-| \chi_{0}-\left.\chi_{0}^{(n)}\right|_{L^{1}(\Omega)} \\
\leqslant & C^{\#} \int_{0}^{t} \int_{\Omega}\left(\left|\theta-\theta^{(n)}\right|+L\left|\chi-\chi^{(n)}\right|\right)(x, s) \mathrm{d} x \mathrm{~d} s \\
& +C^{\#} \int_{0}^{t} \int_{\Omega}\left|f[\chi, \theta]-f\left[\chi, \theta^{(n)}\right]\right|(x, s) \mathrm{d} x \mathrm{~d} s,
\end{aligned}
$$


and Gronwall's argument yields

$$
\begin{aligned}
& \int_{0}^{t} \int_{\Omega}\left|\chi_{t}-\chi_{t}^{(n)}\right|(x, s) \mathrm{d} x \mathrm{~d} s+\int_{\Omega}\left|\chi-\chi^{(n)}\right|(x, t) \mathrm{d} x \\
& \quad \leqslant e^{C^{\#} L t}\left(\left|\chi_{0}-\chi_{0}^{(n)}\right|_{L^{1}(\Omega)}+C^{\#} \int_{0}^{t} \int_{\Omega}\left(\left|\theta-\theta^{(n)}\right|+\left|f[\chi, \theta]-f\left[\chi, \theta^{(n)}\right]\right|\right)(x, s) \mathrm{d} x \mathrm{~d} s\right),
\end{aligned}
$$

which concludes the proof.

\subsection{The maximum principle and Moser iteration}

We derive here an elementary maximum principle result in the case of mixed type boundary conditions. For a fixed final time $T>0$, we consider in $Q_{T}$, for given functions $a: Q_{T} \rightarrow \mathbb{R}$, $u_{\Gamma}: \Sigma_{T} \rightarrow \mathbb{R}, u_{0}: \Omega \rightarrow \mathbb{R}, R: Q_{T} \times \mathbb{R} \rightarrow \mathbb{R}$, the evolution problem

$$
\left\{\begin{array}{l}
\int_{\Omega} a u_{t} w \mathrm{~d} x+\int_{\Omega}\langle\nabla u, \nabla w\rangle \mathrm{d} x+\int_{\partial \Omega} \gamma\left(u-u_{\Gamma}\right) w \mathrm{~d} s=\int_{\Omega} R(x, t, u) w \mathrm{~d} x \quad \forall w \in H^{1} \\
u(x, 0)=u_{0}(x) \quad \text { a.e. }
\end{array}\right.
$$

under the following hypothesis.

HYPOTHESIS 3.5 The data in 3.10 have the following properties:

(i) $a \in L^{\infty}\left(Q_{T}\right), a(x, t) \geqslant a_{*}>0$ a.e.

(ii) $\gamma \in L^{\infty}(\partial \Omega), \gamma \geqslant 0$ a.e.

(iii) $\exists h \in L^{\infty}(0, T):\left|R\left(x, t, u_{1}\right)-R\left(x, t, u_{2}\right)\right| \leqslant h(t)\left|u_{1}-u_{2}\right|$ a.e. $\forall u_{1}, u_{2} \in \mathbb{R}$.

(iv) $R(\cdot, \cdot, 0) \in L^{2}\left(Q_{T}\right), R(x, t, 0) \leqslant 0$ a.e.

(v) $u_{\Gamma} \in L^{2}\left(\Sigma_{T}\right),\left(u_{\Gamma}\right)_{t} \in L^{2}\left(\Sigma_{T}\right), u_{\Gamma} \leqslant 0$ a.e.

(vi) $u_{0} \in H^{1}, u_{0} \leqslant 0$ a.e.

Proposition 3.6 Let Hypothesis 3.5 hold. Then Problem (3.10) admits a unique solution $u \in$ $L^{2}\left(Q_{T}\right)$ such that $u_{t} \in L^{2}\left(Q_{T}\right), \nabla u \in L^{\infty}(0, T ; H)$. Moreover, we have $u(x, t) \leqslant 0$ a.e. in $Q_{T}$.

Sketch of the proof. For a sufficiently large discretization parameter $n \in \mathbb{N}$, we consider the timediscrete problem with time step $\delta=T / n$,

$$
\begin{aligned}
& \frac{1}{\delta} \int_{\Omega} a_{k}\left(u_{k}-u_{k-1}\right) w \mathrm{~d} x+\int_{\Omega}\left\langle\nabla u_{k}, \nabla w\right\rangle \mathrm{d} x+\int_{\partial \Omega} \gamma\left(u_{k}-u_{\Gamma k}\right) w \mathrm{~d} s \\
&=\int_{\Omega} R_{k}\left(x, u_{k}\right) w \mathrm{~d} x \quad \forall w \in H^{1}, k=1, \ldots, n,
\end{aligned}
$$

where $u_{0}$ is defined as in $(3.10)$, and where $a_{k}(x), R_{k}(x, \cdot)$ for $x \in \Omega$, and $u_{\Gamma k}(x)$ for $x \in \partial \Omega$, are the integral means of the corresponding functions in $(3.10)$ over the time interval $[(k-1) \delta, k \delta]$. The existence of $u_{k} \in H^{1}$ is obtained e.g. from the Lax-Milgram lemma, recursively for $k=1, \ldots, n$, whenever $n>T|h|_{\infty} / a_{*}$. Choosing $w=u_{k}^{+}$(the positive part of $u_{k}$ ) in 3.11, and assuming that $u_{k-1} \leqslant 0$ a.e., we obtain

$$
\int_{\Omega} \frac{a_{k}}{\delta}\left|u_{k}^{+}\right|^{2} \mathrm{~d} x+\int_{\Omega}\left|\nabla u_{k}^{+}\right|^{2} \mathrm{~d} x+\int_{\partial \Omega} \gamma\left|u_{k}^{+}\right|^{2} \mathrm{~d} s \leqslant|h|_{\infty} \int_{\Omega}\left|u_{k}^{+}\right|^{2} \mathrm{~d} x .
$$


We have $a_{k}(x) \geqslant a_{*}$, hence $u_{k} \leqslant 0$ a.e. for all $k=1, \ldots, n$. Choosing now $w=u_{k}-u_{k-1}$ in (3.11), we derive in a standard way a priori estimates that enable us to pass to the limit as $n \rightarrow \infty$ and prove the existence of a nonpositive solution to 3.10 . To check that this solution is unique, consider two solutions $u^{1}, u^{2}$ and set $\bar{u}=u^{1}-u^{2}$. We now test the difference of 3.10 , written for $u^{1}$ and $u^{2}$, by a suitable regularization of $\bar{u}_{t}$, Galerkin for instance, choosing basis functions from the complete orthonormal system $\left\{\psi_{k} ; k \in \mathbb{N}\right\}$ in $H$ of eigenfunctions of the problem

$$
-\Delta \psi_{k}=\lambda_{k} \psi_{k} \quad \text { in } \Omega, \quad \partial_{\mathbf{n}} \psi_{k}+\gamma \psi_{k}=0 \quad \text { on } \partial \Omega .
$$

Passing to the limit in the Galerkin approximations, we obtain

$$
a_{*} \int_{0}^{t} \int_{\Omega}\left|\bar{u}_{t}\right|^{2} \mathrm{~d} x \mathrm{~d} \tau \leqslant|h|_{\infty} \int_{0}^{t} \int_{\Omega}\left|\bar{u} \bar{u}_{t}\right| \mathrm{d} x \mathrm{~d} \tau,
$$

hence $\bar{u}=0$ by Gronwall's lemma.

COROLlary 3.7 Let Hypotheses 3.5.i)-(iii) hold, and let (iv)-(vi) be replaced by

(iv) $R(\cdot, \cdot, 0) \in L^{2}\left(Q_{T}\right), R(x, t, 0) \leqslant R^{*}$ a.e.

(v) $u_{\Gamma} \in L^{2}\left(\Sigma_{T}\right),\left(u_{\Gamma}\right)_{t} \in L^{2}\left(\Sigma_{T}\right), u_{\Gamma} \leqslant u_{\Gamma}^{*}$ a.e.

(vi) $u_{0} \in H^{1}, u_{0} \leqslant u^{*}$ a.e.

with some positive constants $R^{*}, u_{\Gamma}^{*}, u^{*}$. Set $K=\max \left\{u^{*}, u_{\Gamma}^{*}\right\}$, consider some constant $B_{1} \geqslant$ $R^{*} / K$, and for $t \in[0, T]$ put

$$
H(t)=\frac{1}{a_{*}} \int_{0}^{t}\left(B_{1}+h(\tau)\right) \mathrm{d} \tau .
$$

Then Problem 3.10 has a unique solution with the regularity from Proposition 3.6 such that

$$
u(x, t) \leqslant K e^{H(t)} \quad \text { a.e. }
$$

Proof. For $(x, t) \in Q_{T}$ and $v \in \mathbb{R}$ set $v_{\Gamma}(x, t)=u_{\Gamma}(x, t)-K e^{H(t)}, \tilde{R}(x, t, v)=R(x, t, v+$ $\left.K e^{H(t)}\right)-K \dot{H}(t) a(x, t) e^{H(t)}$. Then $\tilde{R}$ fulfils the conditions 3.5 (iii)-(iv), since $\tilde{R}(\cdot, \cdot, 0) \in L^{2}\left(Q_{T}\right)$ and

$$
\begin{aligned}
\tilde{R}(x, t, 0) & =R\left(x, t, K e^{H(t)}\right)-K \dot{H}(t) a(x, t) e^{H(t)} \\
& \leqslant R(x, t, 0)+K e^{H(t)}(h(t)-a(x, t) \dot{H}(t)) \\
& \leqslant R^{*}+K e^{H(t)}(h(t)-a(x, t) \dot{H}(t)) \\
& \leqslant\left(1-\frac{a(x, t)}{a_{*}}\right)\left(R^{*}+K h(t) e^{H(t)}\right) \leqslant 0 .
\end{aligned}
$$

A function $v$ on $Q_{T}$ with appropriate regularity is a solution to the equation

$\int_{\Omega} a v_{t} w \mathrm{~d} x+\int_{\Omega}\langle\nabla v, \nabla w\rangle \mathrm{d} x+\int_{\partial \Omega} \gamma\left(v-v_{\Gamma}\right) w \mathrm{~d} s=\int_{\Omega} \tilde{R}(x, t, v) w \mathrm{~d} x \quad \forall w \in H^{1}$

with initial condition $v(x, 0)=v_{0}:=u_{0}(x)-K$ if and only if $u(x, t):=v(x, t)+K e^{H(t)}$ is a solution to Problem 3.10). Since $\tilde{R}, a, \gamma, v_{\Gamma}$, and $v_{0}$ fulfil Hypothesis 3.5. from Proposition 3.6 it follows that $v$ is uniquely determined and $v(x, t) \leqslant 0$ a.e. Hence $u$ is uniquely determined and satisfies the desired growth condition (3.15). 
COROLlary 3.8 Let Hypotheses 3.5(i)-(iii) hold, and let (iv)-(vi) be replaced by

(iv)" $R(\cdot, \cdot, 0) \in L^{2}\left(Q_{T}\right), R(x, t, 0) \geqslant 0$ a.e.

(v)" $u_{\Gamma} \in L^{2}\left(\Sigma_{T}\right),\left(u_{\Gamma}\right)_{t} \in L^{2}\left(\Sigma_{T}\right), u_{\Gamma} \geqslant 0$ a.e.

(vi)" $u_{0} \in H^{1}, u_{0}(x) \geqslant u_{*} \psi_{1}(x)$ a.e.

with some positive constant $u_{*}$, where $\psi_{1}$ is the positive eigenfunction corresponding to the smallest eigenvalue $\lambda_{1} \geqslant 0$ of 3.13 for $k=1$. Consider any constant $B_{2} \geqslant \lambda_{1}$, and for $t \in[0, T]$ put

$$
H(t)=\frac{1}{a_{*}} \int_{0}^{t}\left(B_{2}+h(\tau)\right) \mathrm{d} \tau .
$$

Then Problem 3.10 has a unique solution with the regularity from Proposition 3.6 and such that

$$
u(x, t) \geqslant u_{*} \psi_{1}(x) e^{-H(t)} \quad \text { a.e. }
$$

Proof. For $(x, t) \in Q_{T}$ and $v \in \mathbb{R}$ set $\tilde{R}(x, t, v)=-R\left(x, t,-v+u_{*} \psi_{1}(x) e^{-H(t)}\right)+u_{*} e^{-H(t)}\left(\lambda_{1}-\right.$ $a \dot{H}(t)) \psi_{1}(x)$, and $v_{\Gamma}(x, t)=-u_{\Gamma}(x, t)$. Then $\tilde{R}$ fulfils again the conditions 3.5 (iii)-(iv), since $\tilde{R}(\cdot, \cdot, 0) \in L^{2}\left(Q_{T}\right)$ and

$$
\begin{aligned}
\tilde{R}(x, t, 0) & =-R\left(x, t, u_{*} \psi_{1}(x) e^{-H(t)}\right)+u_{*} e^{-H(t)}\left(\lambda_{1}-a \dot{H}(t)\right) \psi_{1}(x) \\
& \leqslant-R(x, t, 0)+u_{*} \psi_{1}(x) e^{-H(t)}\left(\lambda_{1}+h(t)-a(x, t) \dot{H}(t)\right) \\
& \leqslant u_{*} \psi_{1}(x) e^{-H(t)}\left(1-\frac{a(x, t)}{a_{*}}\right)\left(\lambda_{1}+h(t)\right) \leqslant 0 .
\end{aligned}
$$

As in the proof of Corollary 3.7, we have a one-to-one correspondence between the solution $v$ to 3.16 with initial condition $v(x, 0)=u_{*} \psi_{1}(x)-u_{0}(x)$ and the solution $u(x, t)=u_{*} \psi_{1}(x) e^{-H(t)}-$ $v(x, t)$ to Problem (3.10). By Proposition 3.6 we have again $v \leqslant 0$ a.e., and the assertion immediately follows.

Consider now in $Q_{T}$ the problem

$$
\left\{\begin{array}{l}
\int_{\Omega} a u_{t} w \mathrm{~d} x+\int_{\Omega}\langle\nabla u, \nabla w\rangle \mathrm{d} x+\int_{\partial \Omega} \gamma\left(u-u_{\Gamma}\right) w \mathrm{~d} s \\
\quad=\int_{\Omega}\left(r(x, t)+h_{1}(x, t) u+h_{2}(x, t) u|\log | u||\right) w \mathrm{~d} x \quad \forall w \in H^{1}, \\
u(x, 0)=u_{0}(x) \quad \text { a.e. }
\end{array}\right.
$$

under Hypotheses 3.5 (i)-(ii), where $u_{\Gamma}$ has the regularity as in (v), and with given functions $r, h_{1}, h_{2} \in L^{\infty}\left(Q_{T}\right)$, assuming that

$$
\begin{aligned}
& 0 \leqslant r(x, t) \leqslant r^{*}, \quad 0 \leqslant u_{\Gamma}(x, t) \leqslant u_{\Gamma}^{*}, \\
& \left|h_{i}(x, t)\right| \leqslant h^{*} \quad \text { for } i=1,2, \quad u_{*} \psi_{1}(x) \leqslant u_{0}(x) \leqslant u^{*}
\end{aligned}
$$

a.e. in the respective domains, where $r^{*}, h^{*}, u_{\Gamma}^{*}, u_{*}, u^{*}$ are fixed positive constants. Set

$$
\begin{aligned}
K & =\max \left\{u^{*}, u_{\Gamma}^{*}\right\}, \\
A & =\max \left\{0, \log K,-\log \left(u_{*} \psi_{*}\right)\right\}, \\
B & =\max \left\{\lambda_{1}, r^{*} / K\right\}+(2+A) h^{*}, \\
C & =\frac{1}{a_{*}}\left(B+h^{*}\right),
\end{aligned}
$$

where $\psi_{*}>0$ is a uniform lower bound for $\psi_{1}(x)$. 
Proposition 3.9 Problem 3.17 has a unique solution, and

$$
u_{*} \psi_{1}(x) e^{-H(t)} \leqslant u(x, t) \leqslant K e^{H(t)} \quad \text { a.e. }
$$

where

$$
H(t)=\frac{1}{a_{*}} \int_{0}^{t}\left(B+h^{*} e^{C \tau}\right) \mathrm{d} \tau .
$$

Proof. For all admissible values of the arguments, set

$$
R(x, t, u)=r(x, t)+h_{1}(x, t) u+h_{2}(x, t) u \min \left\{|\log | u||, A+e^{C t}\right\} .
$$

Then $R(x, t, 0)=r(x, t) \in\left[0, r^{*}\right]$, and

$$
\left|\partial_{u} R(x, t, u)\right| \leqslant h^{*}\left(2+A+e^{C t}\right) \quad \text { a.e. }
$$

We may thus apply Corollaries $3.7+3.8$ and conclude that the solution to 3.10$)$ satisfies the estimates 3.19. It remains to check that $u$ is a solution to 3.17. From 3.19) it follows that

$$
\log \left(u_{*} \psi_{*}\right)-H(t) \leqslant \log u(x, t) \leqslant \log K+H(t) \quad \text { a.e., }
$$

hence the constraint $A+e^{C t}$ in 3.20 is never active, and the assertion follows.

Finally, we derive here a global in time Moser-type estimate (cf. [1]) for nonhomogeneous mixed boundary conditions. We follow in principle the scheme of [22], showing in addition the explicit dependence upon some parameters of the problem, which is needed in the proof of Theorem 2.2. We state the result in the space

$$
L_{\mathrm{loc}}^{\infty}\left(Q_{\infty}\right):=\left\{u: \Omega \times(0, \infty) \rightarrow \mathbb{R} ;\left.u\right|_{Q_{T}} \in L^{\infty}\left(Q_{T}\right) \text { for all } T>0\right\} .
$$

We will also make repeated use of the well-known interpolation inequality

$$
|v|_{H} \leqslant A\left(\eta|\nabla v|_{H}+\eta^{-N / 2}|v|_{L^{1}(\Omega)}\right),
$$

which holds for every $v \in H^{1}$ and every $\eta \in(0,1)$, with a positive constant $A$ that depends on $\Omega$, but neither on $v$ nor on $\eta$.

Proposition 3.10 Given a nonnegative function $\gamma \in L^{1}(\partial \Omega)$, consider the problem

$$
\left\{\begin{array}{c}
\int_{\Omega} a(x, t) u_{t} w \mathrm{~d} x+\int_{\Omega}\langle\nabla u, \nabla w\rangle \mathrm{d} x+\int_{\partial \Omega} \gamma(x)\left(h(x, t, u)-u_{\Gamma}(x, t)\right) w \mathrm{~d} s \\
=\int_{\Omega} \mathcal{H}[u] w \mathrm{~d} x \quad \forall w \in H^{1} \\
u(x, 0)=u_{0}(x) \quad \text { a.e. }
\end{array}\right.
$$

under the assumption that there exist positive constants $H_{0}, H_{1}, C_{h}, a_{0}, a_{1}, A_{0}, U, U_{\Gamma}, E_{0}$ such that the following holds:

(i) The mapping $\mathcal{H}: L_{\text {loc }}^{\infty}\left(Q_{\infty}\right) \rightarrow L_{\text {loc }}^{\infty}\left(Q_{\infty}\right)$ has the property that

$$
u(x, t) \mathcal{H}[u](x, t) \leqslant H_{1}|u(x, t)|+H_{0}|u(x, t)|^{2} \quad \text { a.e. in } Q_{\infty}, \forall u \in L_{\mathrm{loc}}^{\infty}\left(Q_{\infty}\right) .
$$


(ii) $h$ is a Carathéodory function on $Q_{\infty} \times \mathbb{R}$ such that $h(x, t, u) u \geqslant C_{h} u^{2}$ a.e. for all $u \in \mathbb{R}$.

(iii) $a, a_{t} \in L^{\infty}\left(Q_{\infty}\right)$ are such that $a_{0} \leqslant a(x, t) \leqslant A_{0}$ and $\left|a_{t}(x, t)\right| \leqslant a_{1}$ a.e. in $Q_{\infty}$.

(iv) $u^{0} \in L^{\infty}(\Omega),\left|u^{0}(x)\right| \leqslant U$ a.e. in $\Omega$.

(v) $u_{\Gamma} \in L^{\infty}\left(\Sigma_{\infty}\right),\left|u_{\Gamma}(x, t)\right| \leqslant U_{\Gamma}$ a.e. on $\Sigma_{\infty}$.

(vi) There exists a solution $u \in L_{\mathrm{loc}}^{\infty}\left(Q_{\infty}\right) \cap L_{\mathrm{loc}}^{2}\left(0, \infty ; H^{1}\right)$ to 3.23 such that $u_{t} \in L_{\mathrm{loc}}^{2}(0, \infty ; H)$, satisfying the a priori estimate

$$
\int_{\Omega}|u(x, t)| \mathrm{d} x \leqslant E_{0} \quad \text { a.e. in }(0, \infty) .
$$

Then there exists a positive constant $C^{*}$ depending only on $A$ (cf. 3.22$)$ ), $|\Omega|,|\gamma|_{L^{1}(\partial \Omega)}, C_{h}, U$, $U_{\Gamma}, a_{0}$, and $A_{0}$ such that

$$
|u(t)|_{L^{\infty}(\Omega)} \leqslant C^{*}\left(1+a_{1}+H_{0}\right)^{1+N / 2}\left(1+H_{1}+E_{0}\right) \quad \text { for a.e. } t>0 .
$$

Proof. We prove Proposition 3.10 under the additional hypothesis

$$
U=U_{\Gamma}=C_{h}=H_{1}=E_{0}=1 .
$$

The general result is then easily obtained via the transformation

$$
\tilde{\gamma}=C_{h} \gamma, \quad \tilde{u}=u / K, \quad K=\max \left\{E_{0}, H_{1}, U, U_{\Gamma} / C_{h}\right\} .
$$

During the proof we will denote by $C_{i}, i=1,2, \ldots$, some positive constants depending only on $A$, $|\Omega|,|\gamma|_{L^{1}(\partial \Omega)}, a_{0}$, and $A_{0}$.

For $k \in \mathbb{N}$, set $w=u|u|^{2^{k}-2}$ in 3.23 . In what follows, we make use of the identity

$$
a(x, t) u_{t} u|u|^{2^{k}-2}=2^{-k} \frac{\partial}{\partial t}\left(a(x, t)|u|^{2^{k}}\right)-2^{-k} a_{t}|u|^{2^{k}},
$$

and of the inequality

$$
\begin{aligned}
\int_{\Omega}\left\langle\nabla u, \nabla\left(u|u|^{2^{k}-2}\right)\right\rangle \mathrm{d} x+ & \int_{\partial \Omega} \gamma\left(h(x, t, u)-u_{\Gamma}\right) u|u|^{2^{k}-2} \mathrm{~d} s \\
& \geqslant \frac{2^{k}-1}{2^{2 k-2}} \int_{\Omega}\left|\nabla\left(u|u|^{2^{k-1}-1}\right)\right|^{2} \mathrm{~d} x+\int_{\partial \Omega} \gamma\left(|u|^{2^{k}}-|u|^{2^{k}-1}\right) \mathrm{d} s .
\end{aligned}
$$

Set $\Phi_{k}=u|u|^{2^{k-1}-1}$. Then we get, using Hölder's and Young's inequalities,

$$
\begin{aligned}
2^{-k} \frac{\mathrm{d}}{\mathrm{d} t} \int_{\Omega} a(x, t)\left|\Phi_{k}\right|^{2} \mathrm{~d} x+ & \frac{2^{k}-1}{2^{2 k-2}} \int_{\Omega}\left|\nabla \Phi_{k}\right|^{2} \mathrm{~d} x+\int_{\partial \Omega} \gamma\left|\Phi_{k}\right|^{2} \mathrm{~d} s \\
\leqslant & \left(\int_{\partial \Omega} \gamma\left|\Phi_{k}\right|^{2} \mathrm{~d} s\right)^{1-2^{-k}}\left(\int_{\partial \Omega} \gamma \mathrm{d} s\right)^{2^{-k}}+a_{1} 2^{-k} \int_{\Omega}\left|\Phi_{k}\right|^{2} \mathrm{~d} x \\
& +\int_{\Omega}\left(H_{0}\left|\Phi_{k}\right|^{2}+\left|\Phi_{k}\right|^{2\left(1-2^{-k}\right)}\right) \mathrm{d} x \\
\leqslant & \left(1-2^{-k}\right) \int_{\partial \Omega} \gamma\left|\Phi_{k}\right|^{2} \mathrm{~d} s+2^{-k} \int_{\partial \Omega} \gamma \mathrm{d} s \\
& +a_{1} 2^{-k} \int_{\Omega}\left|\Phi_{k}\right|^{2} \mathrm{~d} x+\left(H_{0}+1\right) \int_{\Omega}\left|\Phi_{k}\right|^{2} \mathrm{~d} x+2^{-k}|\Omega| .
\end{aligned}
$$


Setting $H_{2}:=1+a_{1}+H_{0}$ and multiplying the above inequality by $2^{k}$, we find that

$$
\frac{\mathrm{d}}{\mathrm{d} t} \int_{\Omega} a(x, t)\left|\Phi_{k}\right|^{2} \mathrm{~d} x+2 \int_{\Omega}\left|\nabla \Phi_{k}\right|^{2} \mathrm{~d} x \leqslant 2^{k} H_{2} \int_{\Omega}\left|\Phi_{k}\right|^{2} \mathrm{~d} x+|\Omega|+\int_{\partial \Omega} \gamma \mathrm{d} s .
$$

We now use the interpolation inequality 3.22 to derive the inequalities

$$
\begin{aligned}
& \int_{\Omega}\left|\Phi_{1}\right|^{2} \mathrm{~d} x \leqslant 2 A^{2}\left(\eta^{2}\left|\nabla \Phi_{1}\right|_{H}^{2}+\eta^{-N} E_{0}^{2}\right) \\
& \int_{\Omega}\left|\Phi_{k}\right|^{2} \mathrm{~d} x \leqslant 2 A^{2}\left(\eta^{2}\left|\nabla \Phi_{k}\right|_{H}^{2}+\eta^{-N}\left|\Phi_{k-1}\right|_{H}^{4}\right) \quad \text { for } k>1 .
\end{aligned}
$$

For $k=1$, we infer from 3.27 and 3.28 that

$$
\begin{aligned}
\frac{\mathrm{d}}{\mathrm{d} t} \int_{\Omega} a(x, t)\left|\Phi_{1}\right|^{2} \mathrm{~d} x+2 \int_{\Omega} & \left|\nabla \Phi_{1}\right|^{2} \mathrm{~d} x \\
& \leqslant 4 H_{2} A^{2}\left(\eta^{2} \int_{\Omega}\left|\nabla \Phi_{1}\right|^{2} \mathrm{~d} x+\eta^{-N} E_{0}^{2}\right)+|\Omega|+\int_{\partial \Omega} \gamma \mathrm{d} s .
\end{aligned}
$$

Choosing $\eta=1 /\left(2 \mathrm{~A} \sqrt{\mathrm{H}_{2}}\right)$, we find that

$$
\frac{\mathrm{d}}{\mathrm{d} t} \int_{\Omega} a(x, t)\left|\Phi_{1}\right|^{2} \mathrm{~d} x+\int_{\Omega}\left|\nabla \Phi_{1}\right|^{2} \mathrm{~d} x \leqslant C_{1} H_{2}^{1+N / 2} .
$$

For $k>1$, we choose $\eta=1 /\left(A \sqrt{2^{k+1} H_{2}}\right)$, we conclude from 3.27) and (3.29) that

$$
\frac{\mathrm{d}}{\mathrm{d} t} \int_{\Omega} a(x, t)\left|\Phi_{k}\right|^{2} \mathrm{~d} x+\left|\nabla \Phi_{k}\right|_{H}^{2} \leqslant C_{2}\left(1+\left(2^{k} H_{2}\right)^{1+N / 2}\left|\Phi_{k-1}\right|_{H}^{4}\right) .
$$

Using again 3.29 with $\eta=1 /(\sqrt{2} A)$, it follows for a.e. $t>0$ that

$$
\begin{aligned}
& \frac{\mathrm{d}}{\mathrm{d} t} \int_{\Omega} a(x, t)\left|\Phi_{1}\right|^{2} \mathrm{~d} x+\left|\Phi_{1}(t)\right|_{H}^{2} \leqslant C_{3} H_{2}^{1+N / 2}, \\
& \frac{\mathrm{d}}{\mathrm{d} t} \int_{\Omega} a(x, t)\left|\Phi_{k}\right|^{2} \mathrm{~d} x+\left|\Phi_{k}(t)\right|_{H}^{2} \leqslant C_{4}\left(1+\left(2^{k} H_{2}\right)^{1+N / 2}\left|\Phi_{k-1}(t)\right|_{H}^{4}\right) .
\end{aligned}
$$

By assumption, we have $a_{0}\left|\Phi_{k}(t)\right|_{H}^{2} \leqslant \int_{\Omega} a(x, t)\left|\Phi_{k}\right|^{2} \mathrm{~d} x \leqslant A_{0}\left|\Phi_{k}(t)\right|_{H}^{2}$, and $\left|\Phi_{k}(0)\right|_{H}^{2} \leqslant|\Omega|$. Hence,

$$
\begin{aligned}
& \left|\Phi_{1}(t)\right|_{H}^{2} \leqslant C_{5} H_{2}^{1+N / 2}, \\
& \left|\Phi_{k}(t)\right|_{H}^{2} \leqslant C_{6}\left(1+\left(2^{k} H_{2}\right)^{1+N / 2} \max _{0 \leqslant \tau \leqslant t}\left|\Phi_{k-1}(\tau)\right|_{H}^{4}\right) .
\end{aligned}
$$

Define now

$$
z_{k}(t)=\max _{0 \leqslant \tau \leqslant t}|u(\tau)|_{L^{2^{k}}(\Omega)}=\max _{0 \leqslant \tau \leqslant t}\left|\Phi_{k}(\tau)\right|_{H}^{2^{-k}}
$$

Then we have

$$
\begin{aligned}
& z_{1}(t) \leqslant C_{7} H_{2}^{(1+N / 2) / 2}, \\
& z_{k}(t) \leqslant C_{8}^{2^{-k}}\left(2^{k} H_{2}\right)^{(1+N / 2) 2^{-k}} \max \left\{1, z_{k-1}(t)\right\} .
\end{aligned}
$$


In particular, putting $y_{k}(t)=\max \left\{1, z_{k}(t)\right\}$, we get

$$
\begin{aligned}
& y_{1}(t) \leqslant C_{9} H_{2}^{(1+N / 2) / 2}, \\
& y_{k}(t) \leqslant\left(C_{10} H_{2}^{(1+N / 2)}\right)^{2^{-k}} 2^{k(1+N / 2) 2^{-k}} y_{k-1}(t) \quad \text { for } k \geqslant 2 .
\end{aligned}
$$

Hence, we conclude that

$$
\begin{aligned}
\log y_{k}(t) & \leqslant \sum_{j=1}^{k} 2^{-j}\left(\log \left(C_{11} H_{2}^{1+N / 2}\right)+j(1+N / 2) \log 2\right) \\
& \leqslant(1+N / 2)\left(C_{12}+\log H_{2}\right)
\end{aligned}
$$

independently of $k$ and $t>0$. Thus, it suffices to choose $C^{*}=\exp \left(C_{12}(1+N / 2)\right)$, and conclude that

$$
\sup _{t \geqslant 0, k \in \mathbb{N}}|u(t)|_{L^{2^{k}}(\Omega)} \leqslant C^{*} H_{2}^{1+N / 2}=C^{*}\left(1+a_{1}+H_{0}\right)^{1+N / 2} .
$$

Formula 3.24 now follows from 3.25 3.26).

\section{Proof of the uniqueness result}

We start with the proof of Theorem 2.4 Relation 2.2 is for (almost) all $x \in \Omega$ of the form (3.4), with

$$
\begin{aligned}
\alpha(\theta) & =\frac{\mu(\theta)}{\beta+\theta}, \\
g=f[\theta, \chi] & =-\frac{1}{\beta+\theta}\left(c_{V}^{\prime}(\chi) \theta(1-\log \theta)+\lambda^{\prime}(\chi)+\theta \sigma^{\prime}(\chi)+b[\chi]\right) .
\end{aligned}
$$

Within the range $\underline{\theta}<\theta<\bar{\theta}$ and $\chi \in \mathcal{D}_{C}(\varphi), \chi_{t} \leqslant C$ of admissible values for the solutions (taking indeed $\underline{\theta}=\min \left\{\underline{\theta}_{1}, \underline{\theta}_{2}\right\}$ etc.), all nonlinearities in $(2.1,2.2)$ are Lipschitz continuous. Using the notation from Theorem 2.4 we obtain, as a consequence of [3.7), for a.e. $(x, t) \in Q_{\infty}$ the estimate

$$
\left|\hat{\chi}_{t}(x, t)\right|+\frac{\partial}{\partial t}|\hat{\chi}(x, t)| \leqslant R_{0}\left(|\hat{\theta}(x, t)|+|\hat{\chi}(x, t)|+\int_{\Omega}|\hat{\chi}(y, t)| \mathrm{d} y\right),
$$

with some constant $R_{0}$. Let us fix some $T>0$. In what follows, we denote by $R_{1}, R_{2}, \ldots$ suitable constants depending possibly on $T$, but independent of the solutions. Integrating (4.1) over $\Omega$, we find by Gronwall's argument that

$$
\int_{\Omega}|\hat{\chi}(y, t)| \mathrm{d} y \leqslant R_{1}\left(\int_{\Omega}\left|\hat{\chi}_{0}(y)\right| \mathrm{d} y+\int_{0}^{t} \int_{\Omega}|\hat{\theta}(y, \tau)| \mathrm{d} y \mathrm{~d} \tau\right) .
$$

Hence, testing 4.1 by $e^{-R_{0} t}$, and using 4.2 , we get

$$
\int_{0}^{t}\left|\hat{\chi}_{t}(x, \tau)\right| \mathrm{d} \tau+|\hat{\chi}(x, t)| \leqslant R_{2}\left(\left|\hat{\chi}_{0}(x)\right|+\int_{0}^{t}|\hat{\theta}(x, \tau)| \mathrm{d} \tau+\int_{0}^{t} \int_{\Omega}|\hat{\theta}(y, \tau)| \mathrm{d} y \mathrm{~d} \tau\right)
$$


for a.e. $x \in \Omega$ and every $t \in[0, T]$. In particular,

$$
\int_{0}^{t} \int_{\Omega}\left|\hat{\chi}_{t}(x, \tau)\right| \mathrm{d} x \mathrm{~d} \tau \leqslant R_{3}\left(\int_{\Omega}\left|\hat{\chi}_{0}(x)\right| \mathrm{d} x+\int_{0}^{t} \int_{\Omega}|\hat{\theta}(x, \tau)| \mathrm{d} x \mathrm{~d} \tau\right) .
$$

We now multiply 4.3 by $|\hat{\chi}(x, t)|$ and integrate over $\Omega$ to get, for all $t \in[0, T]$,

$$
\int_{\Omega}|\hat{\chi}(x, t)|^{2} \mathrm{~d} x \leqslant R_{4}\left(\left|\hat{\chi}_{0}\right|_{H}^{2}+\int_{0}^{t} \int_{\Omega}|\hat{\theta}(x, \tau)|^{2} \mathrm{~d} x \mathrm{~d} \tau\right) .
$$

The crucial point is to exploit 2.9 properly. Notice first that we have

$$
b[\chi] \chi_{t}(x, t)=2 B[\chi]_{t}(x, t)+2 \int_{\Omega} k(x, y) G^{\prime}(\chi(x, t)-\chi(y, t)) \chi_{t}(y, t) \mathrm{d} y .
$$

We integrate the difference of the two equations $(2.9)$, written for $\left(\theta_{1}, \chi_{1}\right)$ and $\left(\theta_{2}, \chi_{2}\right)$, from 0 to $t$, rewriting the terms $b\left[\chi_{i}\right]\left(\chi_{i}\right)_{t}$ according to 4.6 . We test the result by $\hat{\theta}(x, t)$. Using the Lipschitz continuity of all nonlinearities ( $\varphi$ is Lipschitz continuous on $\mathcal{D}_{C}(\varphi)$ with constant $C$ ), and writing $\hat{\Theta}(x, t)=\int_{0}^{t} \hat{\theta}(x, \tau) \mathrm{d} \tau, \hat{\Theta}_{\Gamma}(x, t)=\int_{0}^{t} \hat{\theta}_{\Gamma}(x, \tau) \mathrm{d} \tau$, we infer for each $t>0$ that

$$
\begin{gathered}
c_{0} \int_{\Omega}|\hat{\theta}(x, t)|^{2} \mathrm{~d} x+\frac{\mathrm{d}}{\mathrm{d} t}\left(\frac{1}{2} \int_{\Omega}|\nabla \hat{\Theta}|^{2} \mathrm{~d} x+\frac{1}{2} \int_{\partial \Omega} \gamma \hat{\Theta}^{2} \mathrm{~d} s-\int_{\partial \Omega} \gamma \hat{\Theta} \hat{\Theta}_{\Gamma} \mathrm{d} s\right)+\int_{\partial \Omega} \gamma \hat{\Theta} \hat{\theta}_{\Gamma} \mathrm{d} s \\
\leqslant R_{5}\left(\left|\hat{\theta}_{0}\right|_{H}^{2}+\left|\hat{\chi}_{0}\right|_{H}^{2}+\int_{\Omega}|\hat{\chi}(x, t)|^{2} \mathrm{~d} x+\int_{0}^{t} \int_{\Omega} \int_{\Omega} k(x, y)\left|\hat{\chi}_{t}(y, \tau)\right||\hat{\theta}(x, t)| \mathrm{d} x \mathrm{~d} y \mathrm{~d} \tau\right) .
\end{gathered}
$$

The last term on the right-hand side of the above inequality can be estimated, using (4.4), as

$$
\begin{array}{r}
\int_{0}^{t} \int_{\Omega} \int_{\Omega} k(x, y)\left|\hat{\chi}_{t}(y, \tau)\right||\hat{\theta}(x, t)| \mathrm{d} x \mathrm{~d} y \mathrm{~d} \tau \leqslant R_{6} \int_{\Omega}|\hat{\theta}(x, t)| \mathrm{d} x \int_{0}^{t} \int_{\Omega}\left|\hat{\chi}_{t}(y, \tau)\right| d y \mathrm{~d} \tau \\
\leqslant R_{7}\left(\int_{\Omega}|\hat{\theta}(x, t)|^{2} \mathrm{~d} x\right)^{1 / 2}\left(\int_{\Omega}\left|\hat{\chi}_{0}(x)\right|^{2} \mathrm{~d} x+\int_{0}^{t} \int_{\Omega}|\hat{\theta}(x, \tau)|^{2} \mathrm{~d} x \mathrm{~d} \tau\right)^{1 / 2} .
\end{array}
$$

Combining the last two inequalities again with the Gronwall lemma, we obtain for each $t \in[0, T]$ the estimate

$$
\begin{aligned}
& \int_{0}^{t} \int_{\Omega}|\hat{\theta}(x, \tau)|^{2} \mathrm{~d} x \mathrm{~d} \tau+\int_{\Omega}|\nabla \hat{\Theta}(x, t)|^{2} \mathrm{~d} x+\int_{\partial \Omega} \gamma \hat{\Theta}^{2}(s, t) \mathrm{d} s \\
& \quad \leqslant R_{8}\left(\left|\hat{\theta}_{0}\right|_{H}^{2}+\left|\hat{\chi}_{0}\right|_{H}^{2}+\int_{0}^{t} \int_{\partial \Omega} \gamma \hat{\theta}_{\Gamma}^{2}(s, \tau) \mathrm{d} s \mathrm{~d} \tau+\int_{0}^{t} \int_{\Omega}|\hat{\chi}(x, \tau)|^{2} \mathrm{~d} x \mathrm{~d} \tau\right) .
\end{aligned}
$$

We now multiply 4.7 by $2 R_{4}$, add the result to 4.5, and see that Gronwall's argument can be applied again to arrive at the final estimate

$$
\int_{\Omega}|\hat{\chi}(x, t)|^{2} \mathrm{~d} x+\int_{0}^{t} \int_{\Omega}|\hat{\theta}(x, \tau)|^{2} \mathrm{~d} x \mathrm{~d} \tau \leqslant R_{8}\left(\left|\hat{\theta}_{0}\right|_{H}^{2}+\left|\hat{\chi}_{0}\right|_{H}^{2}+\int_{0}^{t} \int_{\partial \Omega} \gamma \hat{\theta}_{\Gamma}^{2}(s, \tau) \mathrm{d} s \mathrm{~d} \tau\right) .
$$

With this, Theorem 2.4 is proved. 


\section{Proof of the existence result}

This section is devoted to the proof of Theorem 2.2 (i.e. the existence of weak solutions to system (2.1 2.4 , 2.6 2.8). We use a standard technique: we first truncate system $2.1,2.2$, prove existence of solutions to the truncated problem, and finally show that the solution of this system is also a solution of the original system, removing the truncation.

Assuming Hypothesis 2.1 to hold, we proceed as follows: first solve the problem corresponding to $2.1-2.4$, in which we regularize the coefficient $\mu$ and the logarithmic contribution in 2.2), replace $\theta$ by $|\theta|$ at suitable places, and then derive upper and lower bounds for $\theta$ that will allow us to conclude that the solution of the modified problem satisfies also $2.1-2.4,2.6-2.8$. For some sufficiently large cut-off parameter $\varrho>1$, which will be specified later, we introduce for $\theta \in \mathbb{R}$ the functions

$$
\begin{aligned}
& \mu_{\varrho}(\theta)= \begin{cases}\mu(|\theta|) & \text { for }|\theta| \leqslant \varrho, \\
\mu(\varrho)+\mu_{*}(|\theta|-\varrho) & \text { for }|\theta|>\varrho,\end{cases} \\
& L_{\varrho}(\theta)= \begin{cases}0 & \text { for } \theta \leqslant 0, \\
\log \theta & \text { for } 0<\theta<\varrho, \\
\log \varrho & \text { for } \theta \geqslant \varrho,\end{cases}
\end{aligned}
$$

and consider the following problem:

PRoBlem 5.1 For $T>0$ find a pair $(\theta, \chi)$ with the regularity 2.6 and 2.8 restricted to the time interval $[0, T]$, solving a.e. in $Q_{T}$ the system of equations

$$
\begin{aligned}
& \left(c_{V}(\chi) \theta+\lambda(\chi)+\beta \varphi(\chi)\right)_{t}+b[\chi] \chi_{t}-\Delta \theta=0, \\
& \mu_{\varrho}(\theta) \chi_{t}+c_{V}^{\prime}(\chi) \theta\left(1-L_{\varrho}(\theta)\right)+\lambda^{\prime}(\chi)+\theta \sigma^{\prime}(\chi)+(\beta+|\theta|) \partial \varphi(\chi)+b[\chi] \ni 0,
\end{aligned}
$$

with boundary and initial conditions 2.32 .4 , in the sense of Theorem 2.2

Lemma 5.2 For each fixed $\varrho>1$, there exists at least one solution to Problem 5.1. Moreover, there exist positive numbers $c_{\varrho, T}<C_{\varrho, T}$ such that $c_{\varrho, T} \leqslant \theta(x, t) \leqslant C_{\varrho, T}$ a.e.

Proof. Consider the Faedo-Galerkin approximations

$$
\theta^{m}(x, t)=\sum_{k=1}^{m} \theta_{k}(t) \psi_{k}(x),
$$

where $\left\{\psi_{k} ; k \in \mathbb{N}\right\}$ is the complete orthonormal system in $H$ of eigenfunctions of the problem (3.13), and where the $\theta_{k}(t)$ satisfy the system of equations

$$
\begin{aligned}
& \int_{\Omega}\left(c_{V}(\chi) \theta^{m}\right)_{t} \psi_{k} \mathrm{~d} x+\int_{\Omega}\left\langle\nabla \theta^{m}, \nabla \psi_{k}\right\rangle \mathrm{d} x+\int_{\partial \Omega} \gamma\left(\theta^{m}-\theta_{\Gamma}\right) \psi_{k} \mathrm{~d} s \\
&=-\int_{\Omega}\left((\lambda(\chi)+\beta \varphi(\chi))_{t}+b[\chi] \chi_{t}\right) \psi_{k} \mathrm{~d} x, \quad k=1, \ldots, m, \\
& \mu_{\varrho}\left(\theta^{m}\right) \chi_{t}+c_{V}^{\prime}(\chi) \theta^{m}\left(1-L_{\varrho}\left(\theta^{m}\right)\right)+\lambda^{\prime}(\chi)+\theta \sigma^{\prime}(\chi)+\left(\beta+\left|\theta^{m}\right|\right) \partial \varphi(\chi)+b[\chi] \ni 0,
\end{aligned}
$$

with initial conditions

$$
\begin{aligned}
\theta_{k}(0) & =\int_{\Omega} \theta_{0} \psi_{k} \mathrm{~d} x, \\
\chi(x, 0) & =\chi_{0}(x) .
\end{aligned}
$$


It follows from Proposition 3.2 and Remark 3.3 that 5.6 defines a mapping that with each $\theta^{m} \in$ $L^{1}\left(Q_{T}\right)$ associates continuously $\chi, \chi_{t}$ and $\varphi(\chi)_{t}$ in any $L^{p}\left(Q_{T}\right)$. Equation 5.5 therefore has the form

$$
\int_{\Omega} F\left[\theta^{m}\right] \theta_{t}^{m} \psi_{k} \mathrm{~d} x+\lambda_{k} \theta_{k}=\int_{\partial \Omega} \gamma \theta_{\Gamma} \psi_{k} \mathrm{~d} s+\int_{\Omega} H\left[\theta^{m}\right] \psi_{k} \mathrm{~d} x,
$$

with continuous operators $F, H: L^{1}\left(Q_{T}\right) \rightarrow L^{p}\left(Q_{T}\right)$ for suitably chosen $p>1$, and such that $\tilde{C}_{1} \geqslant F[\theta](x, t) \geqslant c_{0},|H[\theta](x, t)| \leqslant \tilde{C}_{1}$ a.e. for all $\theta \in L^{1}\left(Q_{T}\right)$ with a constant $\tilde{C}_{1}>0$ independent of $\theta$. The matrix $A_{j k}[\theta](t)=\int_{\Omega} F[\theta](x, t) \psi_{j}(x) \psi_{k}(x) \mathrm{d} x$ is symmetric and positive definite, hence Lipschitz continuous solutions $\theta_{1}(t), \ldots, \theta_{m}(t)$ to (5.9), 5.7) are well defined on $[0, T]$. Testing 5.9 by $\dot{\theta}_{k}$, and summing over $k$, yields

$$
\begin{aligned}
\int_{\Omega} F\left[\theta^{m}\right]\left|\theta_{t}^{m}\right|^{2} \mathrm{~d} x+\frac{\mathrm{d}}{\mathrm{d} t}\left(\frac{1}{2} \int_{\Omega}\left|\nabla \theta^{m}\right|^{2} \mathrm{~d} x+\frac{1}{2} \int_{\partial \Omega} \gamma\left|\theta^{m}\right|^{2} \mathrm{~d} s-\int_{\partial \Omega} \gamma \theta_{\Gamma} \theta^{m} \mathrm{~d} s\right) \\
=-\int_{\partial \Omega} \gamma\left(\theta_{\Gamma}\right)_{t} \theta^{m} \mathrm{~d} s+\int_{\Omega} H\left[\theta^{m}\right] \theta_{t}^{m} \mathrm{~d} x \\
\leqslant\left(\int_{\partial \Omega} \gamma\left|\left(\theta_{\Gamma}\right)_{t}\right|^{2} \mathrm{~d} s\right)^{1 / 2}\left(\int_{\partial \Omega} \gamma\left|\theta^{m}\right|^{2} \mathrm{~d} s\right)^{1 / 2}+\tilde{C}_{2}\left(\int_{\Omega}\left|\theta_{t}^{m}\right|^{2} \mathrm{~d} x\right)^{1 / 2} .
\end{aligned}
$$

Integrating from 0 to $t \in(0, T]$, we obtain from Gronwall's argument the estimate

$$
\left|\theta_{t}^{m}\right|_{L^{2}\left(Q_{T}\right)}+\left|\nabla \theta^{m}\right|_{L^{\infty}(0, T ; H)}+\sup _{[0, T]} \int_{\partial \Omega} \gamma\left|\theta^{m}\right|^{2} \mathrm{~d} s \leqslant \tilde{C}_{3},
$$

with constants $\tilde{C}_{2}, \tilde{C}_{3}>0$ independent of $m$ (depending possibly on $T$, but $T$ is kept fixed here). Selecting a subsequence if necessary, we may pass to the limit in $5.5+5.6$ as $m \rightarrow \infty$ to obtain the existence result.

To derive the bounds for $\theta$, we first estimate $\chi_{t}$ using Proposition 3.2 and (5.4), taking into account 3.3. Note that the term $\left|\theta\left(1-L_{\varrho}(\theta)\right)\right|$ is bounded from above independently of $t$ by a constant multiple of $1+|\theta|(1+\log \varrho)$. We thus deduce the following bound on the $\chi$-component of the solution $(\theta, \chi)$ to Problem 5.1 .

$$
\left|\chi_{t}\right|_{L^{\infty}\left(Q_{T}\right)}+\left|\varphi(\chi)_{t}\right|_{L^{\infty}\left(Q_{T}\right)} \leqslant c_{1}(1+\log \varrho)^{2} .
$$

Here and below, $c_{1}, c_{2}, \ldots$ denote constants independent of $\varrho$ and $T$. Having still in mind weak solutions in the sense of Theorem 2.2, we rewrite 5.3 formally as

$$
c_{V}(\chi) \theta_{t}-\Delta \theta=\mu_{\varrho}(\theta) \chi_{t}^{2}-c_{V}^{\prime}(\chi) \chi_{t} \theta L_{\varrho}(\theta)+\theta \sigma(\chi)_{t}+|\theta| \varphi(\chi)_{t} .
$$

We are thus in the situation of $3.17+3.18$ ) with the choice $u=\theta$, and

$$
\begin{aligned}
& a(x, t)=c_{V}(\chi), \quad r(x, t)=\frac{\mu_{\varrho}(\theta)}{1+|\theta|} \chi_{t}^{2}, \\
& h_{1}(x, t)=\sigma(\chi)_{t}+\operatorname{sign}(\theta)\left(\varphi(\chi)_{t}+\frac{\mu_{\varrho}(\theta)}{1+|\theta|} \chi_{t}^{2}\right), \\
& h_{2}(x, t)=-c_{V}^{\prime}(\chi) \chi_{t} \frac{L_{\varrho}(\theta)}{|\log | \theta||}, \\
& u_{\Gamma}=\theta_{\Gamma}, \quad u^{0}=\theta_{0}, \quad u_{*}=\theta_{*} / \psi^{*},
\end{aligned}
$$

and the upper and lower bounds for $\theta$ follow from Proposition 3.9 
REMARK 5.3 If we examine the proof more closely, we see that the hypothesis on $\left(\theta_{\Gamma}\right)_{t}$ can be relaxed using the trace theorem for functions from $H^{1}$ for $N \geqslant 2$. The argument still works for $\left(\theta_{\Gamma}\right)_{t} \in L^{2}\left(0, T ; L^{p}(\partial \Omega)\right)$ with $p \geqslant 2(N-1) / N$, or $\left(\theta_{\Gamma}\right)_{t} \in L^{2}\left(0, T ; H^{-1 / 2}(\partial \Omega)\right)$, if $\partial \Omega$ is smooth.

The uniqueness and continuous dependence result in Theorem 2.4 holds indeed for problem (5.3 5.4 , 2.3 2.4) as well. We can therefore extend the solution to (5.3 5.4), 2.3 2.4) to the whole time interval $[0, \infty)$ and obtain the following result.

COROLlary 5.4 There exists a solution to (5.3), 5.4), $2.3,2.4$ in the sense of Theorem 2.2 on $Q_{\infty}$ with the properties $2.7, \sqrt{2.8}$, and functions $\theta_{\varrho}^{l}, \theta_{\varrho}^{u}:(0, \infty) \rightarrow(0, \infty)$ such that $\theta_{\varrho}^{l}$ is nonincreasing, $\theta_{\varrho}^{u}(T)$ is nondecreasing, and $\theta_{\varrho}^{l}(T) \leqslant \theta(x, t) \leqslant \theta_{\varrho}^{u}(T)$ for a.e. $(x, t) \in Q_{T}$ and for all $T>0$.

We see in particular that we can remove the absolute values in 5.3 5.4). Our aim is now to prove that the solution to Problem 5.1 satisfies also $(2.1+2.4),(2.6-2.8)$ for suitably chosen $\varrho$. To this end, we derive a uniform upper bound for $\theta$. Then, choosing $\varrho$ above this bound, we will check that the solution to 5.355 .4 is the desired solution to $2.1-2.2$.

Equation (5.3) is of the form as in Proposition 3.10, with

$$
\begin{gathered}
u=\theta, \quad a(x, t)=c_{V}(\chi), \quad h(x, t, u)=u, \\
\mathcal{H}[u]=-(\lambda(\chi)+\beta \varphi(\chi))_{t}-b[\chi] \chi_{t}-c_{V}^{\prime}(\chi) \chi_{t} u .
\end{gathered}
$$

Referring to Proposition 3.10 we have $a_{0}=c_{0}, C_{h}=1$, and $U+U_{\Gamma}+A_{0} \leqslant c_{2}$. The other parameters, however, namely $a_{1}, H_{0}, H_{1}$, and $E_{0}$, do depend on $\varrho$ by 5.12$)$. Hypothesis 2.1 and (5.12) yield

$$
a_{1}+H_{0}+H_{1} \leqslant c_{3}(1+\log \varrho)^{2} .
$$

It remains to determine the dependence of $E_{0}$ on $\varrho$. To do so, we test 5.3 by $\psi_{1}$ from 2.5 . This yields

$$
\begin{aligned}
\frac{\mathrm{d}}{\mathrm{d} t} \int_{\Omega} c_{V}(\chi) \theta \psi_{1} \mathrm{~d} x+\int_{\Omega}\left\langle\nabla \theta, \nabla \psi_{1}\right\rangle \mathrm{d} x & +\int_{\partial \Omega} \gamma\left(\theta-\theta_{\Gamma}\right) \psi_{1} \mathrm{~d} s \\
& =-\int_{\Omega}\left((\lambda(\chi)+\beta \varphi(\chi))_{t}+b[\chi] \chi_{t}\right) \psi_{1} \mathrm{~d} x .
\end{aligned}
$$

If $\gamma \equiv 0$, we may take $\psi_{1} \equiv 1$, and using the symmetry of $B[\chi]$, we deduce from (5.17p that

$$
\begin{aligned}
\int_{\Omega} c_{V}(\chi) \theta(x, t) \mathrm{d} x= & \int_{\Omega} c_{V}\left(\chi_{0}\right) \theta_{0} \mathrm{~d} x+\int_{\Omega}\left(\lambda\left(\chi_{0}\right)+\beta \varphi\left(\chi_{0}\right)+B\left[\chi_{0}\right]\right) \mathrm{d} x \\
& -\int_{\Omega}(\lambda(\chi)+\beta \varphi(\chi)+B[\chi])(x, t) \mathrm{d} x \\
\leqslant & c_{4} .
\end{aligned}
$$

Assume now that $\int_{\partial \Omega} \gamma \mathrm{d} s>0$. Then $\lambda_{1}>0$, and we have

$$
\frac{\mathrm{d}}{\mathrm{d} t} \int_{\Omega} c_{V}(\chi) \theta \psi_{1} \mathrm{~d} x+\lambda_{1} \int_{\Omega} \theta \psi_{1} \mathrm{~d} x=\int_{\partial \Omega} \gamma \theta_{\Gamma} \psi_{1} \mathrm{~d} s-\int_{\Omega}\left((\lambda(\chi)+\beta \varphi(\chi))_{t}+b[\chi] \chi_{t}\right) \psi_{1} \mathrm{~d} x .
$$


From Hypothesis 2.1 and estimate 5.12 , we infer that

$$
\frac{\mathrm{d}}{\mathrm{d} t} \int_{\Omega} c_{V}(\chi) \theta \psi_{1} \mathrm{~d} x+c_{5} \int_{\Omega} c_{V}(\chi) \theta \psi_{1} \mathrm{~d} x \leqslant c_{6}(1+\log \varrho)^{2} .
$$

Hence, $\int_{\Omega} \theta \psi_{1} \mathrm{~d} x \leqslant c_{7}(1+\log \varrho)^{2}$, and using Hypothesis 2.1 (ii), we obtain the final estimate

$$
E_{0} \leqslant c_{8}(1+\log \varrho)^{2} .
$$

Referring to 3.24) in Proposition 3.10, we find that

$$
\theta(x, t) \leqslant c_{9}(1+\log \varrho)^{4+N}
$$

for a.e. $(x, t) \in Q_{\infty}$. Taking now any $\varrho$ such that

$$
\varrho>c_{9}(1+\log \varrho)^{4+N},
$$

we see that the solution to Problem 5.1 is also a solution to $2.1-2.4$, $2.6-2.8$, and the upper bound in 2.10$)$ is satisfied. It remains to derive the uniform (in time) lower bound in 2.10 ). To this end, let us consider the function

$$
z=\log \bar{\theta}-\log \theta>0 \quad \text { a.e. in } Q_{\infty},
$$

with $\bar{\theta}$ defined in 2.10). Using 2.2, we rewrite 2.9) as

$$
\begin{aligned}
& \int_{\Omega} c_{V}(\chi) \theta_{t} w \mathrm{~d} x+\int_{\Omega}\langle\nabla \theta, \nabla w\rangle \mathrm{d} x+\int_{\partial \Omega} \gamma\left(\theta-\theta_{\Gamma}\right) w \mathrm{~d} s \\
&=\int_{\Omega}\left(\mu(\theta) \chi_{t}^{2}-c_{V}^{\prime}(\chi) \chi_{t} \theta \log \theta+\theta \sigma(\chi)_{t}+\theta \varphi(\chi)_{t}\right) w \mathrm{~d} x .
\end{aligned}
$$

We now choose $w=v / \theta$ with $v \in H^{1}$ to obtain

$$
\begin{aligned}
\int_{\Omega} c_{V}(\chi) \frac{\theta_{t}}{\theta} v \mathrm{~d} x & +\int_{\Omega} \frac{1}{\theta}\langle\nabla \theta, \nabla v\rangle \mathrm{d} x+\int_{\partial \Omega} \gamma\left(1-\frac{\theta_{\Gamma}}{\theta}\right) v \mathrm{~d} s \\
& =\int_{\Omega}\left(\frac{|\nabla \theta|^{2}}{\theta^{2}}+\frac{\mu(\theta)}{\theta} \chi_{t}^{2}-c_{V}^{\prime}(\chi) \chi_{t} \log \theta+\sigma(\chi)_{t}+\varphi(\chi)_{t}\right) v \mathrm{~d} x
\end{aligned}
$$

In terms of $z$, we have

$$
\begin{aligned}
\int_{\Omega}\left(c_{V}(\chi) z\right)_{t} v \mathrm{~d} x+\int_{\Omega}\langle\nabla z, \nabla v\rangle \mathrm{d} x & +\int_{\partial \Omega} \gamma\left(\frac{\theta_{\Gamma}}{\bar{\theta}} e^{z}-1\right) v \mathrm{~d} s \\
& =-\int_{\Omega}\left(\frac{|\nabla \theta|^{2}}{\theta^{2}}+\frac{\mu(\theta)}{\theta} \chi_{t}^{2}+(\sigma(\chi)+\varphi(\chi))_{t}\right) v \mathrm{~d} x .
\end{aligned}
$$

We are thus again in the situation of Proposition 3.10 with $h(x, t, u)=\left(\theta_{\Gamma} / \bar{\theta}\right)\left(e^{u}-1\right)$ suitably extended for $u<0$, and it only remains to find a uniform $L^{1}$-bound for $z$ as in Proposition 3.10.(vi). We proceed as above and test (5.24) by $\psi_{1}$. This yields

$$
\frac{\mathrm{d}}{\mathrm{d} t} \int_{\Omega}\left(c_{V}(\chi) z+\varphi(\chi)+\sigma(\chi)\right) \psi_{1} \mathrm{~d} x+\lambda_{1} \int_{\Omega} z \psi_{1} \mathrm{~d} x+\int_{\partial \Omega} \gamma\left(\frac{\theta_{\Gamma}}{\bar{\theta}} e^{z}-1-z\right) \psi_{1} \mathrm{~d} s \leqslant 0
$$


The case $\gamma \equiv 0, \lambda_{1}=0$ is again straightforward. For $\lambda_{1}>0$ we notice that $\left(\theta_{\Gamma} / \bar{\theta}\right) e^{z}-1-z \geqslant-c_{10}$, hence a uniform bound for $z$ in $L^{1}(\Omega)$ follows again from the uniform Gronwall lemma. From Proposition 3.10 we conclude that

$$
z(x, t) \leqslant c_{11} \quad \text { a.e. in } Q_{\infty} .
$$

Hence, $\theta(x, t) \geqslant \bar{\theta} e^{-c_{11}}$ a.e., which completes the proof of Theorem 2.2 .

\section{REFERENCES}

1. Alikakos, N. D. $L^{p}$-bounds of solutions of reaction-diffusion equations. Comm. Partial Differential Equations 4 (1979), 827-868. Zbl 0421.35009 MR 0537465

2. Bates, P., \& ChmaJ, A. An integrodifferential model for phase transitions: stationary solutions in higher space dimensions. J. Statist. Phys. 95 (1999), 1119-1139. Zbl 0958.82015 MR 1712445

3. BAtes, P., \& HAN, J. The Neumann boundary problem for a nonlocal Cahn-Hilliard equation. J. Differential Equations 212 (2005), 235-277. Zbl 1081.45004 MR 2129092

4. Bers, L., John, F., \& Schechter, M. Partial Differential Equations. Wiley (1964). Zbl 0126.00207 MR 0163043

5. BREZIS, H. Opérateurs maximaux monotones et semi-groupes de contractions dans les espaces de Hilbert. North-Holland Math. Stud. 5, North-Holland, Amsterdam (1973). Zbl 0252.47055 MR 0348562

6. Brokate, M., \& Sprekels, J. Hysteresis and Phase Transitions. Appl. Math. Sci. 121, Springer, New York (1996). Zbl 0951.74002 MR 1411908

7. Caginalp, G. An analysis of a phase field model of a free boundary. Arch. Ration. Mech. Anal. 92 (1986), 205-245. Zbl 0608.35080 MR 0816623

8. CAHn, J., \& Hilliard, J. Free energy of a nonuniform system. I. Interfacial free energy. J. Chem. Phys. 28 (1958), 258-267.

9. Chen, C. K., \& FIfe, P. C. Nonlocal models of phase transitions in solids. Adv. Math. Sci. Appl. 10 (2000), 821-849. Zbl 0977.74044 MR 1807453

10. Colli, P., Krejčí, P., Rocca, E., \& Sprekels, J. Nonlinear evolution inclusions arising from phase change models. Czechoslovak Math. J., to appear.

11. Feireisl, E., Issard-Roch, F., \& Petzeltová, H. A non-smooth version of the Łojasiewicz-Simon theorem with applications to nonlocal phase-field systems. J. Differential Equations 199 (2004), 1-21. Zbl 1062.35152 MR 2041509

12. GaJewski, H. On a nonlocal model of non-isothermal phase separation. Adv. Math. Sci. Appl. 12 (2002), 569-586. Zbl 1039.80001 MR 1943981

13. Gajewski, H., \& Griepentrog, J. A. A descent method for the free energy of multicomponent systems. Discrete Contin. Dyn. Syst. 15 (2006), 505-528. MR 2199441

14. Gajewski, H., \& Zacharias, K. On a nonlocal phase separation model. J. Math. Anal. Appl. 286 (2003), 11-31. Zbl 1032.35078 MR 2009615

15. Giacomin, G., \& Lebowitz, J. L. Phase segregation dynamics in particle systems with long range interactions. I. Macroscopic limits. J. Statist. Phys. 87 (1997), 37-61. Zbl $0937.82037 \mid$ MR 1453735

16. Giacomin, G., \& Lebowitz, J. L. Phase segregation dynamics in particle systems with long range interactions. II. Interface motion. SIAM J. Appl. Math. 58 (1998), 1707-1729. Zbl 1015.82027 MR 1638739

17. Grasselli, M., Petzeltová, H., \& Schimperna, G. A nonlocal phase-field system with inertial term. Quart. Appl. Math., to appear. 
18. Griepentrog, J. A. On the unique solvability of a nonlocal phase separation problem for multicomponent systems. Nonlocal Elliptic and Parabolic Problems, Banach Center Publ. 66, Inst. Math., Polish. Acad. Sci. (2004), 153-164. Zbl pre02122832 MR 2143362

19. KrejČí, P., RocCA, E., \& Sprekels, J. Nonlocal temperature-dependent phase-field models for nonisothermal phase transitions. J. London Math. Soc., to appear.

20. KRejČí, P., \& Sprekels, J. Nonlocal phase-field models for non-isothermal phase transitions and hysteresis. Adv. Math. Sci. Appl. 14 (2004), 593-612. Zbl 1073.74042 MR 2111831

21. KREJČí, P., \& SPREKELS, J. Long time behaviour of a singular phase transitions model. Discrete Contin. Dyn. Syst. A 15 (2006), 1119-1135. MR 2224500

22. Krejčí, P., Sprekels, J., \& Zheng, S. Asymptotic behaviour for a phase-field system with hysteresis. J. Differential Equations 175 (2001), 88-107. Zbl 1021.35131 MR 1849225

23. KREJČí, P., \& Zheng, S. Pointwise asymptotic convergence of solutions for a phase separation model. Discrete Contin. Dyn. Syst. 16 (2006), 1-18. Zbl 1105.80008 MR 2221733

24. Lions, J.-L., \& MAgenes, E. Problèmes aux limites non homogènes et applications. Vol. 1, Dunod, Paris (1968). Zbl 0165.10801 MR 0247244

25. Penrose, O., \& Fife, P. C. Thermodynamically consistent models of phase field type for the kinetics of phase transitions. Phys. D 43 (1990), 44-62. Zbl 0709.76001 MR 1060043

26. Sprekels, J., \& Zheng, S. Global existence and asymptotic behaviour for a nonlocal phase-field model for non-isothermal phase transitions. J. Math. Anal. Appl. 279 (2003), 97-110. Zbl 1023.35051 MR 1970493

27. Visintin, A. Models of Phase Transitions. Birkhäuser, Boston (1996). Zbl 0882.35004 MR 1423808

28. VAN DER WAALS, J. D. The thermodynamic theory of capillarity flow under the hypothesis of a continuous variation in density. Verhandelingen der Koninklijke Nederlandsche Akademie van Wetenshappen te Amsterdam 1 (1893), 1-56. 